Homology, Homotopy and Applications, vol.21(1), 2019, pp.129-159

\title{
HIGHER-TWISTED PERIODIC SMOOTH DELIGNE COHOMOLOGY
}

\author{
DANIEL GRADY AND HISHAM SATI
}

(communicated by Jonathan M. Rosenberg)

\begin{abstract}
Generalizing degree one twisting of Deligne cohomology, as a differential refinement of integral cohomology, from previous work, here we consider higher degree twists. The Rham complex, hence de Rham cohomology, admits twists of any odd degree. However, in order to consider twists of integral cohomology we need a periodic version. Combining the periodic versions of both ingredients leads us to introduce a periodic form of Deligne cohomology. We demonstrate that this theory indeed admits a twist by a gerbe of any odd degree. We present the main properties of the new theory and illustrate its use with examples and computations, mainly via a corresponding twisted differential Atiyah-Hirzebruch spectral sequence.
\end{abstract}

\section{Introduction}

There has been a lot of recent activity on modifying generalized cohomology theories to include twists and geometric refinements, in order to account for automorphisms and include geometric data. Twisted differential generalized cohomology theories are established at the general axiomatic level [15]. However, working out these theories explicitly is in practice not a straightforward task. Twisting the simplest case of a differential cohomology theory, namely Deligne cohomology, proved to be nontrivial [44] and is closely related to taking coefficients in variations of mixed Hodge structures (see $[\mathbf{1 9}, 45]$ ). Even at the topological level, while twisting of generalized cohomology theories is axiomatically well-established $[62,2,1]$, spelling out explicit constructions requires considerable work (see $[1,72,54]$ for recent illustrations). The goal of this paper is to generalize the degree one twists of Deligne cohomology from [44] to include twists of higher degrees. These will be in the form of higher gerbes, or $n$-bundles, with connections (see $[29,71,74,25,27]$ for constructions and related applications).

Deligne cohomology (see $[22,6,37,51,24,35]$ ) is a differential refinement of ordinary, i.e. integral, cohomology. As such it has various realizations [22, 21, 35, 9, 23, 50, 14, 5, 74], which are (expected to be) equivalent (see [75, 17]). Consider

Received March 28, 2018, revised June 21, 2018, July 13, 2018; published on September 5, 2018. 2010 Mathematics Subject Classification: 14F43, 53C08, 19L50, 18G40, 55R20, 14D23.

Key words and phrases: Deligne cohomology, differential cohomology, gerbe, twisted cohomology, stack, Atiyah-Hirzebruch spectral sequence.

Article available at http://dx.doi.org/10.4310/HHA.2019.v21.n1.a7

Copyright (C) 2018, International Press. Permission to copy for private use granted. 
the sheaf of positively graded chain complexes associated with real-valued differential forms ${ }^{1}$

$$
\mathcal{D}(n):=\left(\cdots \longrightarrow 0 \longrightarrow \underline{\mathbb{Z}} \longrightarrow \Omega^{0} \stackrel{d}{\longrightarrow} \Omega^{1} \stackrel{d}{\longrightarrow} \cdots \stackrel{d}{\longrightarrow} \Omega^{n-1}\right),
$$

where we place differential $(n-1)$-forms in degree 0 and locally constant integervalued functions in degree $n$. Given a smooth manifold $M$, the Deligne cohomology group of degree $n$ is defined to be the sheaf (hyper)cohomology group ${ }^{2} \widehat{H}^{n}(M ; \mathbb{Z}):=$ $H^{0}(M ; \mathcal{D}(n))$. Cech resolutions allow for explicit calculation of these groups. If $\left\{U_{\alpha}\right\}$ is a good open cover of $M$, then one can form the Čech-Deligne double complex (see $[8,9])$. The operator on the totalization of the double complex is the Cech-Deligne operator $D:=d+(-1)^{p} \delta$, with $d$ and $\delta$ the de Rham and Cech differentials, respectively, acting on elements of total degree $p$. The sheaf cohomology group $H^{0}(M ; \mathcal{D}(n))$ can then be identified with the group of diagonal elements $\eta_{k, k}$ in the double complex which are $D$-closed, $D \eta_{k, k}=0$, modulo those which are $D$-exact.

At a more general level, and from a homotopy theory point of view, given a spectrum $E$ the canonical data for the corresponding differential theory is comprised of the following (see [13, Example 4.49]). Let $\mathcal{H}$ be the Eilenberg-MacLane functor and take $A:=\pi_{*} E \otimes \mathbb{R}$ the 'realified' coefficients of the theory. Let $c: E \rightarrow \mathcal{H}(A)$ be the map uniquely determined up to homotopy such that it induces the map 'realifying' the coefficients $\pi_{*}(E) \rightarrow \pi_{*}(A) \cong \pi_{*}(E) \otimes \mathbb{R}, x \mapsto x \otimes 1$. Indeed, for $E=\mathcal{H}(\mathbb{Z})$ the integral Eilenberg-MacLane spectrum, $A=\mathbb{Z} \otimes \mathbb{R} \cong \mathbb{R}$, and $c: \mathcal{H}(\mathbb{Z}) \rightarrow \mathcal{H}(\mathbb{R})$ uniquely determined by $\mathbb{Z} \rightarrow \mathbb{R} \cong \mathbb{Z} \otimes \mathbb{R}, x \mapsto x \otimes 1$. This data determines a differential extension of $\mathcal{H} \mathbb{Z}$, which in degree $n$ takes the form $\left(\Sigma^{n} \mathcal{H} \mathbb{Z}, \mathbb{R}, c\right)$. Applying the Eilenberg-MacLane functor $\mathcal{H}$ to the Deligne complex $\mathcal{D}(n)$ from expression (1.1) gives a natural equivalence of differential spectra (see [13])

$$
\mathcal{H}(\mathcal{D}(n)) \cong\left(\Sigma^{n} \mathcal{H} \mathbb{Z}, \mathbb{R}, c\right) .
$$

Which degrees should the twists $H$ or $\hat{h}$ have? The twists of the Deligne complex, a priori naturally arise in degree one [44]. Note that for the underlying topological theory, a representation of the fundamental group $\pi_{1}(X)$ of a space $X$ on $\operatorname{Aut}(\mathbb{Z}) \cong$ $\mathbb{Z} / 2$ gives $\mathbb{Z}$ the structure of a module over the group ring $\mathbb{Z}\left[\pi_{1}(X)\right]$, which is used in [12] to describe $\pi_{1}(X)$-twisted integral cohomology. On the other hand, one can twist the de Rham complex by differential forms of any odd degree, not just degree one (see $[65,7,76,68,69,59])$. At first glance, this might appear to give an inherent incompatibility of twisted de Rham cohomology and twisted integral cohomology. However, if one takes a closer look, one realizes that twisted de Rham cohomology is really about $\mathbb{Z} / 2$-graded, periodic de Rham cohomology. Thus, one does not expect compatibility with integral cohomology, but rather with periodic integral cohomology. Hence we consider twists of the latter theory in Section 2. This then paves part of the way for us to go towards a general twisted Deligne cohomology. However, as both ingredients, namely de Rham and integral cohomology, were made periodic, we define a periodic version of Deligne cohomology in Section 3. We characterize its main

\footnotetext{
${ }^{1}$ This is sometimes also denoted $\mathbb{Z}_{\mathcal{D}}^{\infty}(n)$ or $\mathbb{Z}(n){ }_{\mathcal{D}}^{\infty}$. We are in the smooth setting throughout, so we will not need extra decorations.

${ }^{2}$ This would be $H^{n}(M ; \mathcal{D}(n))$ if we use the opposite convention. However, the one we use is positively graded, hence better adapted for stacks.
} 
properties via sheaf cohomology and differential spectra, including the ring structure arising from the Deligne-Beilinson cup product $[\mathbf{2 2}, 6]$ (see [25, 27]). Periodic integral differential cohomology groups $\widehat{H}\left(X ; \mathbb{Z}\left[u, u^{-1}\right]\right)$ have been considered from an index theoretic point of view briefly in [55], [34, Sec. 8.4].

We discuss the twists of periodic Deligne cohomology in Section 4. We approach twisting of periodic Deligne cohomology using simplical presheaves and smooth stacks $[29,25,49,27,74]$, as we did in [44]. This approach is very well-suited to the higher twists and allows for the use of powerful algebraic machinery. We will show that the twists indeed refine the twists of both integral cohomology and the de Rham complex. Smooth stacks will arise naturally in twisting periodic Deligne cohomology. Just as we can twist periodic integral cohomology by odd degree singular cocycles (Section 2), we will see that periodic Deligne cohomology can be twisted by higher gerbes of odd degree (Section 4). The appearance of gerbes naturally leads us into the world of smooth stacks, and we will find it useful to recall some of the constructions in this setting (see $[9,29,25,27])$. This requires us to understand in detail exactly what we mean by twisting a periodic differential cohomology theory. We give a characterization of the twists via moduli stacks of higher bundles with connections.

In twisting Deligne cohomology by gerbes of odd degree, it is interesting to see where the gerbe data appears. In fact, as observed in [15], a crucial ingredient in defining twisted differential theories is the analogue of the de Rham isomorphism theorem for twisted cohomology. In the untwisted case, recall that the locally constant sheaf $\underline{\mathbb{R}}$ admits an acyclic resolution via the de Rham complex

$$
\underline{\mathbb{R}} \longleftrightarrow \Omega^{0} \stackrel{d}{\longrightarrow} \Omega^{1} \stackrel{d}{\longrightarrow} \Omega^{2} \longrightarrow \cdots,
$$

and the de Rham Theorem is manifestly a corollary of this fact. Indeed, for a smooth manifold $M$, the sheaf cohomology $H^{*}(M, \underline{\mathbb{R}})$ can be calculated both as Cech cohomology and via this resolution. The isomorphism between singular and Čech cohomology then recovers de Rham's classical theorem.

Just as multiplicative cohomology theories have topological spaces of twists (the Picard spaces) $[60,62,2]$, differential refinements of such theories have smooth stacks of twists. Indeed, the stack of twists $\widehat{\mathrm{Tw}}_{\widehat{\mathcal{R}}}$ for any differentially refined cohomology theory $\widehat{\mathcal{R}}=(\mathcal{R}, c, A)$ was introduced in [15]. This was defined by the pullback ${ }^{3}$ (in the notation of $[43])$

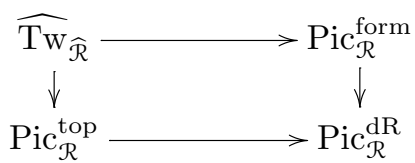

- where $\operatorname{Pic}_{\mathcal{R}}^{\text {top }}$ is the ordinary Picard $\infty$-groupoid of twists for the ring spectrum $\mathcal{R}$, embedded as a constant smooth stack,

- $\operatorname{Pic}_{\mathcal{R}}^{\mathrm{dR}}$ is the Picard stack of sheaves of invertible module spectra over the smash product $\mathcal{R} \wedge \mathcal{H} \mathbb{R}$ (embedded as a constant sheaf of spectra), and

\footnotetext{
${ }^{3}$ We will be dealing with $(\infty, 1)$-categories, so that whenever we talk about pullbacks, pushouts, or any other universal construction, we mean it in the $(\infty, 1)$-sense, i.e., up to higher coherence homotopy.
} 
- $\operatorname{Pic}_{\mathcal{R}}^{\text {form }}$ is the smooth stack which (after evaluation on a smooth manifold $M$ ) comes as the nerve of the groupoid whose objects are weakly locally constant, $\mathrm{K}$-flat, invertible modules over $\left.\Omega^{*}(-; A)\right|_{M}$ (see [15] for details).

An element of the pullback $(1.2)$ can be identified with a triple $\widehat{\mathcal{R}}_{\hat{\tau}}=\left(\mathcal{R}_{\tau}, t, \mathcal{L}\right)$, where $\mathcal{R}_{\tau}$ is an underlying twisted cohomology theory with a topological twist $\tau, \mathcal{L}$ is an invertible module over $\Omega^{*}(-; A)$ and $t$ is an equivalence

$$
t: \mathcal{R}_{\tau} \wedge \mathcal{H} \mathbb{R} \stackrel{\simeq}{\longrightarrow} \mathcal{H}(\mathcal{L})
$$

exhibiting a twisted de Rham theorem. This stack will be important in identifying the twists for periodic Deligne cohomology in Section 4. The situation is summarized in the following tables; first on the untwisted case:

\begin{tabular}{|c||c|c|}
\hline Untwisted cohomology & Ordinary & Periodic \\
\hline \hline Underlying theory & Locally constant sheaf $\underline{\underline{R}}$ & Sheaf of graded alg. $\mathbb{R}\left[u, u^{-1}\right]$ \\
\hline de Rham complex & Ordinary de Rham complex $\Omega^{*}$ & Periodic complex $\Omega^{*}\left[u, u^{-1}\right]$ \\
\hline
\end{tabular}

In [43] we highlighted the close analogies between twisted spectra and line bundles, in that twisted differential spectra are closely related to bundles of spectra equipped with a flat connection. Replacing with twisted analogues:

\begin{tabular}{|c||c|c|}
\hline Twisted cohomology & Ordinary & Periodic \\
\hline \hline Underlying theory & Locally constant sheaf $\mathcal{L}$ & Sheaf of DGA-modules $\mathcal{L} \bullet$ \\
\hline Twist degree & One & Any odd degree \\
\hline Geom. twisting object & Line bundle w. flat conn. $d+H_{1}$ & Gerbe with curvature $H_{2 k+1}$ \\
\hline de Rham complex & $\left(\Omega^{*} \otimes \mathcal{L}, d+H_{1} \wedge\right)$ & $\left(\Omega^{*} \otimes \mathcal{L} \bullet, d+H_{2 k+1} \wedge\right)$ \\
\hline
\end{tabular}

The theories that we consider are related schematically as follows:

$$
\begin{aligned}
& \text { Periodic Deligne } \widehat{H}^{*}\left(M ; \mathcal{D}\left[u, u^{-1}\right]\right) \underset{\text { periodicity }}{\text { forget }} \text { Deligne } \widehat{H}^{*}(M ; \mathbb{Z})=H^{0}(M ; \mathcal{D}(*)) \\
& \left.\begin{array}{c}
\text { forget } \\
\text { connection }
\end{array} \quad \begin{array}{c}
\text { forget } \\
\text { connection }
\end{array}\right\} \\
& \text { Periodic integral } H^{*}\left(M ; \mathbb{Z}\left[u, u^{-1}\right]\right) \sim \underset{\text { periodicity }}{\text { forget }} \longrightarrow \operatorname{Integral~} H^{*}(M ; \mathbb{Z})
\end{aligned}
$$

where the top row, bottom row, left column and right column represent geometric theories, topological theories, periodic theories, and non-periodic theories, respectively. The relations between the corresponding spaces of twists are in turn summarized in the schematic diagram

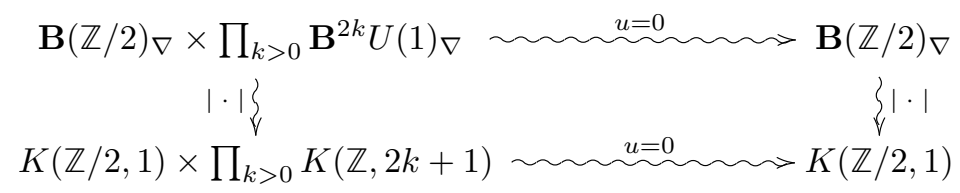

Here $|\cdot|$ is geometric realization, which reduces a geometric theory down to the corresponding topological theory, and $\mathbf{B}(\mathbb{Z} / 2)_{\nabla}$ is the stack of twists for Deligne cohomology [44]. To twist the theories displayed in the first schematic diagram (1.3) one would consider maps from the manifold $M$ to the corresponding space of twists in the second schematic diagram (1.4). 
Explicit Čech cocycles for Deligne cohomology are described in $[35,10,11,39]$. While we do not do this in full generality in the twisted case, we do explain how the Čech cocycle data appear as part of the trivializing data for twisted periodic Deligne cohomology in Section 4.1 (see Lemmas 4.6, 4.7, and 4.8). This involves Chern-Simons type trivialization of Čech-Deligne cocycles, packaged succinctly as in [43]. Extensive discussions of such trivializations relating to Chern-Simons theory can be found in $[10,11,38,32,20,29,78,36,26,70,27,28,77]$. In contrast, cocycles arising from chain complexes would involve an abstract higher local system resulting from the twists of periodic integral cohomology. While this is doable, it does not make the description any more transparent in comparison to the description via spectra; hence we do not consider it in this paper.

Making use of the general constructions in $[42,43]$, we then consider the AtiyahHirzebruch spectral sequence for twisted periodic integral cohomology as well as for twisted periodic Deligne cohomology in Section 5. We provide explicit constructions and characterizations in Section 5.1 and then illustrate the computations via examples in Section 5.2.

We note that there are other approaches to studying the Čech-de Rham double complex. Explicit description of cocycles via the cohomology of the total operator $D$ of the double complex is provided in [63]. Using the notion of Cheeger-Simons cochain sparks [21], a homological machine for the study of secondary geometric invariants called spark complexes is described in $[47,46]$. This seems to be an appropriate setting for twisting differential cohomology in its incarnation as differential characters. While we do not address this, we expect that the resulting twisted versions would be equivalent; our approach places the complication in the coefficients of the (hyper)cohomology while that more homologically flavored approach would place it in the cycles, e.g., via spark complexes.

\section{Twisted periodic integral cohomology}

In this section, we describe the twisted periodic cohomology with both real and integral coefficients. This generalizes twists of integral cohomology [60], also described in modern categorical terms in [1] and geometrically in [31]. This will be a precursor for the de Rham theorem needed to define twisted Deligne cohomology. Throughout the remainder of this paper, we will follow the $\infty$-categorical treatment of twisted cohomology theories $[\mathbf{1}, \mathbf{2}, 72]$ and their differential refinements $[\mathbf{1 5}, 43]$.

\subsection{Twists via bundles of spectra}

The starting point for periodic integral cohomology is the differential graded algebra (DGA) $\mathbb{Z}\left[u, u^{-1}\right]$, equipped with the trivial differential. There is a functor

$$
\mathcal{H}: \mathrm{Ch} \longrightarrow \mathrm{Sp}
$$

from the category of unbounded chain complexes to the category of spectra, called the Eilenberg-MacLane functor. This functor was defined in [73], where it was shown to exhibit an equivalence between $\mathcal{H} \mathbb{Z}$-module spectra and differentially graded $\mathbb{Z}$ algebras. Applying $\mathcal{H}$ to $\mathbb{Z}\left[u, u^{-1}\right]$ we get a spectrum $\mathcal{H} \mathbb{Z}\left[u, u^{-1}\right]$ which represents 
periodic integral cohomology, in the sense that

$$
H^{*}\left(X ; \mathbb{Z}\left[u, u^{-1}\right]\right) \cong H^{*}(X ; \mathbb{Z})\left[u, u^{-1}\right],
$$

where the right hand side is the graded algebra whose elements are formal Laurent polynomials with coefficients in $H^{*}(X ; \mathbb{Z})$ graded by homogeneous degree. The ring structure on the right is induced from the cup product structure on $H^{*}(X ; \mathbb{Z})$, while on the left it is induced from the algebra structure on $\mathbb{Z}\left[u, u^{-1}\right]$. This theory is naturally $\mathbb{Z} / 2$-graded, as we have canonical isomorphisms $H^{*}\left(X ; \mathbb{Z}\left[u, u^{-1}\right]\right) \cong$ $H^{*+2}\left(X ; \mathbb{Z}\left[u, u^{-1}\right]\right)$. For this reason, we will usually refer to the degree of a class as either even or odd.

Remark 2.1 (Action of units on periodic integral cohomology). Being an $\mathcal{H} \mathbb{Z}$-module spectrum, the spectrum $\mathcal{H} \mathbb{Z}\left[u, u^{-1}\right]$ receives an action by $\mathcal{H} \mathbb{Z}$. This action manifests itself simply by the action of the cup product in integral cohomology. More precisely, we have an obvious commutative diagram of unbounded chain complexes

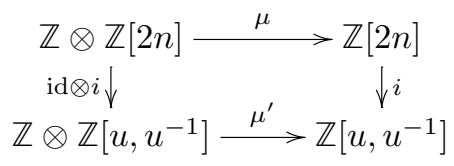

where $\mu: \mathbb{Z} \otimes \mathbb{Z}[2 n] \rightarrow \mathbb{Z}$ is induced by the standard multiplication of integers, $\tilde{\mu}$ is the left unitor map for the monoidal structure and $i_{n}: \mathbb{Z}[2 n] \hookrightarrow \mathbb{Z}\left[u, u^{-1}\right]$ is the canonical map sending the generator $1 \in \mathbb{Z}[2 n]$, in degree $2 n$, to $u^{n} \in \mathbb{Z}\left[u, u^{-1}\right]$. The map $\mu$ induces the cup product operation in integral cohomology

$$
\mathcal{H}(\mu): \mathcal{H} \mathbb{Z} \wedge \Sigma^{2 n} \mathcal{H} \mathbb{Z} \longrightarrow \Sigma^{2 n} \mathcal{H} \mathbb{Z} .
$$

We have a canonical equivalence of spectra $\mathcal{H} \mathbb{Z}\left[u, u^{-1}\right] \simeq \bigvee_{k} \Sigma^{2 k} \mathcal{H} \mathbb{Z}$ and, by the commutativity of Diagram (2.1), $\mathcal{H} \mathbb{Z}$ acts on each factor by the cup product operation.

We now characterize the space of twists of periodic integral cohomology, the first summand of which is the space of twists in the non-periodic case described in [44]. For an $A_{\infty}$-ring spectrum $\mathcal{R}$, defined in the classical sense [61], the grouplike $A_{\infty}$ space of units can be defined as the pullback

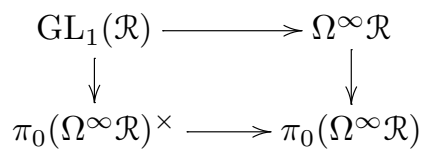

More modern presentations of the symmetric monoidal model category of spectra have a well defined space of automorphisms which agree ${ }^{4}$ with the classical space $\mathrm{GL}_{1}(\mathcal{R})$ (see [2, Proposition 2.3]). The classifying space of twists for a ring spectrum is given by the space $B \mathrm{GL}_{1}(\mathcal{R})$. For $\mathcal{R}=H \mathbb{Z}\left[u, u^{-1}\right]$, we have the following.

Proposition 2.2 (Space of twists for periodic integral cohomology). The space of twists for periodic integral cohomology is

$$
B \mathrm{GL}_{1}\left(\mathcal{H} \mathbb{Z}\left[u, u^{-1}\right]\right) \simeq K(\mathbb{Z} / 2,1) \times \prod_{k>0} K(\mathbb{Z}, 2 k+1) .
$$

${ }^{4}$ In the sense that there is a zig-zag of weak equivalences between the two. 
Proof. We will show that we have an equivalence of grouplike $A_{\infty}$-spaces

$$
\mathbb{Z} / 2 \times \prod_{k>0} K(\mathbb{Z}, 2 k) \simeq \mathrm{GL}_{1}\left(\mathcal{H} \mathbb{Z}\left[u, u^{-1}\right]\right) .
$$

The connected cover of $\mathcal{H} \mathbb{Z}\left[u, u^{-1}\right]$ is given by $\mathcal{H} \mathbb{Z}[u]$ and the infinite loop space is the Dold-Kan image of the positively graded complex $\mathbb{Z}[u] \cong \prod_{k} \mathbb{Z}[2 k]$, which is a model for $\prod_{k} K(\mathbb{Z}, 2 k)$. Since the group of units of $\mathbb{Z}$ are $\mathbb{Z} / 2 \cong\{-1,1\}$, we see that $\mathrm{GL}_{1}\left(\mathcal{H} \mathbb{Z}\left[u, u^{-1}\right]\right)$ is as claimed and delooping gives the desired equivalence.

We now describe the twists via module spectra. In what follows we fix a symmetric monoidal, $\infty$-category of spectra (for example, the presentation by symmetric spectra). For a ring spectrum $\mathcal{R}$, let us recall the Picard $\infty$-groupoid $\mathrm{Pic}_{\mathcal{R}}^{\text {top }}$ from [43], following [15]. This is the infinity groupoid whose objects are invertible $\mathcal{R}$ module spectra. The corresponding geometric realization decomposes in the category of spaces as

$$
\left|\operatorname{Pic}_{\mathcal{R}}^{\text {top }}\right| \simeq B \mathrm{GL}_{1}(\mathcal{R}) \times \pi_{0} \operatorname{Pic}_{\mathcal{R}}^{\text {top }}
$$

For the spectrum $\mathcal{H} \mathbb{Z}\left[u, u^{-1}\right]$, Proposition 2.2 then gives a canonical map

$$
K(\mathbb{Z} / 2,1) \times \prod_{k>0} K(\mathbb{Z}, 2 k+1) \longleftrightarrow\left|\mathrm{Pic}_{\mathcal{H} \mathbb{Z}\left[u, u^{-1}\right]}^{\mathrm{top}}\right|
$$

given by the inclusion at the identity component of the Picard space. This indeed allows us to twist periodic integral cohomology by any odd degree integral class $(\mathbb{Z} / 2$-class in deg. 1$)$.

We now would like to describe the actual module spectra which exhibit the twisted theory. One of the most systematic ways to describe the resulting module spectra was presented in [43]. There, we defined a canonical bundle of spectra over the Picard $\infty$ groupoid which lives in the tangent $\infty$-topos $T$ (Space) (see [57] and [58, section 7.3]). The pullbacks of this universal bundle by a map $h: X \rightarrow \operatorname{Pic}_{\mathcal{R}}^{\text {top }}$ gave a bundle of spectra representing the twisted theory. Since we would like to be as concrete as possible, and relying on as little abstract machinery as possible, we note that in the present case this universal bundle will take on a relatively simple form; see the map (2.3).

In the absence of any geometry, bundles of spectra behave more like covering spaces than like smooth vector bundles. The next example illustrates this point.

Example 2.3 (Bundle of spectra over the circle). Let $Z \rightarrow S^{1}$ be the disconnected cover of $S^{1}$, splitting as the disjoint union $Z=\coprod_{k} W$, where $W$ is the connected cover classified by the subgroup $2 \mathbb{Z} \subset \mathbb{Z} \cong \pi_{1}\left(S^{1}\right)$. This cover can be viewed as a $\mathbb{Z}$ subbundle of the Möbius bundle given by restricting to integers. Viewing $S^{1}$ as the unit circle in the complex plane and removing the points -1 and 1 from $S^{1}$, we get corresponding open sets $U$ and $V$, respectively, covering $S^{1}$. Over $U$ and $V$, we have equivalences

$$
\phi_{U}:\left.Z\right|_{U} \simeq \mathbb{Z} \times U \quad \text { and } \quad \phi_{V}:\left.Z\right|_{V} \simeq \mathbb{Z} \times V,
$$

which can be chosen so that the transition functions act by multiplication by -1 on the fibers, i.e., $\phi_{U V}(n, x)=(-n, x)$. The map $-1 \times: \mathbb{Z} \rightarrow \mathbb{Z}$ extends to a map 
$-1 \times: \mathcal{H}\left(\mathbb{Z}\left[u, u^{-1}\right]\right) \rightarrow \mathcal{H}\left(\mathbb{Z}\left[u, u^{-1}\right]\right)$ degreewise. Gluing by this automorphism gives

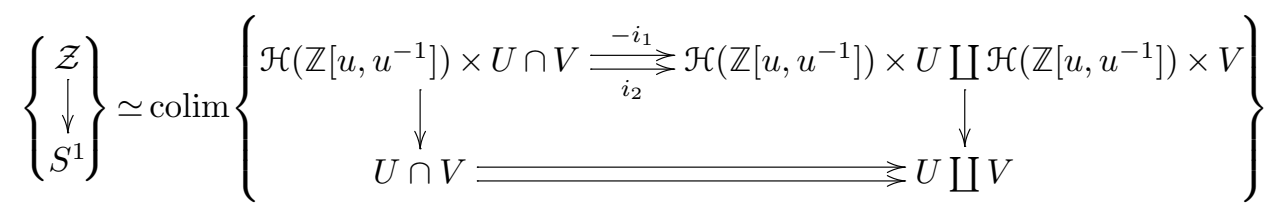

where $i_{2}$ is induced by the usual inclusion into the second factor and the top map $-i_{1}$ applies the automorphism -1 and then includes into the first factor. This colimit takes place in the category $T$ (Space). As part of the data of the colimit, we have local trivializations

$$
\phi_{U}:\left.\mathcal{Z}\right|_{U} \simeq \mathcal{H}\left(\mathbb{Z}\left[u, u^{-1}\right]\right) \times U \quad \text { and } \quad \phi_{V}:\left.\mathcal{Z}\right|_{V} \simeq \mathcal{H}\left(\mathbb{Z}\left[u, u^{-}\right]\right) \times V,
$$

turning $\mathcal{Z}$ into a corresponding bundle of spectra, with fiber $\mathcal{H}\left(\mathbb{Z}\left[u, u^{-1}\right]\right)$, over $S^{1}$. The transition functions take the form $\phi_{U V}(x,-)=-1$, where -1 is the automorphism of the fiber $\mathcal{H}\left(\mathbb{Z}\left[u, u^{-1}\right]\right)$ induced by multiplication by -1 .

In Example 2.3, the automorphisms $\phi_{U V}(x,-)$ had degree zero, in that they were genuine 1-morphisms and not higher simplices in the space of automorphisms $\mathrm{GL}_{1}\left(\mathcal{H}\left(\mathbb{Z}\left[u, u^{-1}\right)\right)\right.$. Now we give an instance where we $d o$ have higher simplices.

Example 2.4 (Bundle of spectra over the 3-sphere). Consider the 3-sphere $S^{3}$, equipped with the cover $\{U, V\}$ obtained by removing the north and south poles, respectively. The intersection $U \cap V \simeq S^{2}$ and, given our identification of the units in (the proof of) Proposition 2.2, we have

$$
\pi_{2}\left(\mathrm{GL}_{1}\left(\mathcal{H} \mathbb{Z}\left[u, u^{-1}\right]\right)\right) \simeq \pi_{2}(K(\mathbb{Z}, 2)) \simeq \mathbb{Z},
$$

with generator $u$. Then the homotopy class of a map $U \cap V \simeq S^{2} \rightarrow \operatorname{GL}_{1}\left(\mathcal{H} \mathbb{Z}\left[u, u^{-1}\right]\right)$ is represented by an integer $n$ times the generator $u$. Via the action of $\mathrm{GL}_{1}(\mathcal{H} \mathbb{Z}[u$, $\left.u^{-1}\right]$ ), such a representative gives rise to a map

$$
n u: S^{2} \longrightarrow \operatorname{Map}\left(\mathcal{H} \mathbb{Z}\left[u, u^{-1}\right], \mathcal{H} \mathbb{Z}\left[u, u^{-1}\right]\right),
$$

and we would like to take the map as supplying the transition data for a bundle on $S^{3}$. Acting by this map and then by the usual inclusion map $U \cap V \hookrightarrow U \coprod V$ into the second factor gives the two top arrows (that is, $\times n u i_{1}$ and $i_{2}$ ), respectively, in

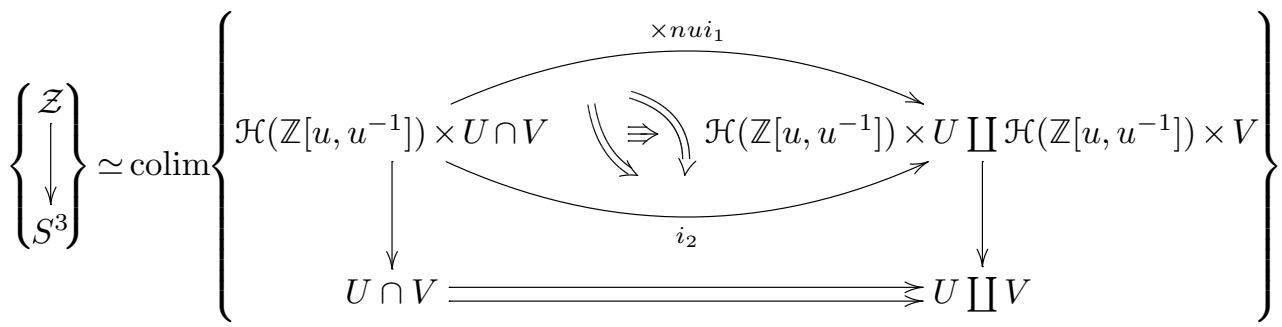

The fact that this diagram has nontrivial homotopies filling it, i.e., the ones provided by the map (2.2), is what separates it from Example 2.3. The homotopy class of sections of the bundle $\mathcal{Z} \rightarrow S^{3}$ computes the twisted cohomology groups.

In the same way that ordinary vector bundles with $G$-structure are classified by maps to the classifying space $B G$, bundles of spectra with fiber $\mathcal{R}$ are classified by 
maps to $B \mathrm{GL}_{1}(\mathcal{R})$. There is a universal bundle of spectra over this space. In the present case (i.e. for periodic integral cohomology) it takes the following form. The action of each factor $K(\mathbb{Z}, 2 k)$ on the spectrum $\mathcal{H} \mathbb{Z}\left[u, u^{-1}\right]$ gives rise to a quotient ${ }^{5}$ $\mathcal{H} \mathbb{Z}\left[u, u^{-1}\right] / / K(\mathbb{Z}, 2 k)$. This leads to the following bundle

$$
\mathcal{H} \mathbb{Z}\left[u, u^{-1}\right] / / K(\mathbb{Z}, 2 k) \longrightarrow K(\mathbb{Z}, 2 k+1),
$$

which we can think of as a universal bundle. Given a map $h: X \rightarrow K(\mathbb{Z}, 2 k+1)$, we consider the pullback diagram

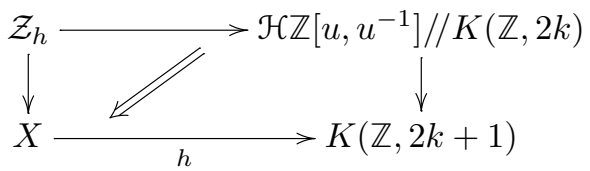

Then $\mathcal{Z}_{h} \rightarrow X$ is itself a bundle of spectra with fiber $\mathcal{H} \mathbb{Z}\left[u, u^{-1}\right]$. Indeed, the Pasting Lemma for pullbacks implies that we have a double pullback square

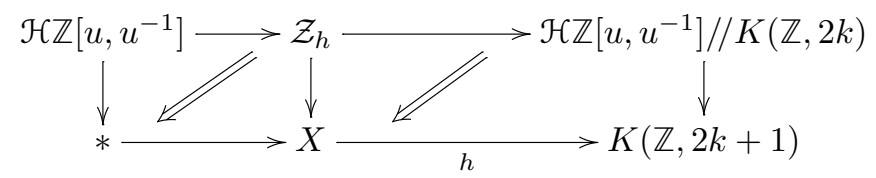

so that $\mathcal{H} \mathbb{Z}\left[u, u^{-1}\right]$ is identified as the fiber. Next, suppose that $X$ admits a good open cover $\left\{U_{\alpha}\right\}$. Then, by the Borsuk Nerve Theorem (see, e.g., [64, Theorem 3.21]), $X$ is homotopy equivalent to the colimit over the Cech nerve of a good open cover $\left\{U_{\alpha}\right\}$. By iterating pullbacks, we therefore get induced homotopy commutative simplicial diagrams

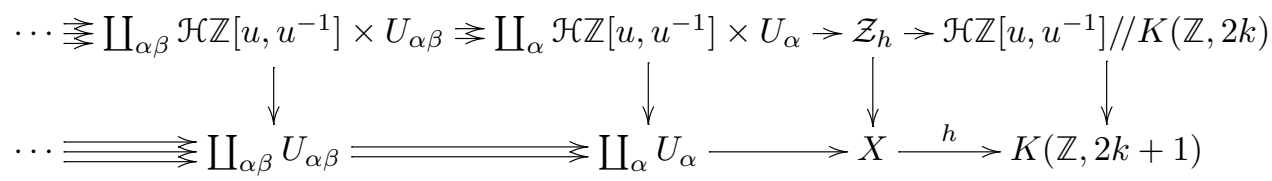

where the bottom simplicial diagram is induced by the Cech nerve and there are chosen homotopies filling the diagram (which we have left implicit). Via the axiom of descent ${ }^{6}$ the top simplicial diagram in (2.4) is homotopy colimiting and this says that (up to homotopy equivalence) we can recover the total space $\mathcal{Z}_{h}$ by gluing together local trivializations via compatibility maps defined on various intersections. This association gives the following correspondence.

Proposition 2.5 (Characterization of twisted periodic integral cohomology). There is a bijective correspondence between homotopy classes of maps $h: X \rightarrow K(\mathbb{Z}, 2 k+1)$ and equivalence classes of bundles of spectra with fiber $\mathcal{H} \mathbb{Z}\left[u, u^{-1}\right]$, which admit a

\footnotetext{
${ }^{5}$ Since we are in an $(\infty, 1)$-category, quotients are taken in the $(\infty, 1)$-sense, i.e., up to coherence homotopy.

${ }^{6}$ The tangent $\infty$-category of spaces is an example of an $\infty$-topos and such infinity categories are characterized axiomatically via the Giraud-Rezk-Lurie axioms [56, Sec. 6.1.5]. One of these axioms is that of descent, which asserts that whenever we have a diagram of the above form with the bottom simplicial diagram being colimiting, and all squares being Cartesian, then the top simplicial diagram is also colimiting.
} 
$K(\mathbb{Z}, 2 k)$-structure, i.e., a reduction of the structure $\infty$-group from $\mathrm{GL}_{1}\left(\mathcal{H} \mathbb{Z}\left[u, u^{-1}\right]\right)$ to $K(\mathbb{Z}, 2 k)$.

Example 2.6 (Classifying map for bundles of spectra over $S^{3}$.). In Example 2.4 the transition data specified by the map $\times n u: S^{2} \simeq U \cap V \rightarrow K(\mathbb{Z}, 2) \hookrightarrow \mathrm{GL}_{1}(\mathcal{H} \mathbb{Z}[u$, $\left.\left.u^{-1}\right]\right)$ corresponds to a map $h: S^{3} \rightarrow K(\mathbb{Z}, 3) \hookrightarrow B \mathrm{GL}_{1}\left(\mathcal{H} \mathbb{Z}\left[u, u^{-1}\right]\right)$ by the loopsuspension adjunction. This map is the classifying map of the bundle constructed there.

Note that the sections of the map $p: \mathcal{Z}_{h} \rightarrow X$ form a spectrum. Given that, locally, $\mathcal{Z}_{h}$ trivializes as $\mathcal{H} \mathbb{Z}\left[u, u^{-1}\right] \times U_{\alpha}$ when $\left\{U_{\alpha}\right\}$ is a good open cover of a space $X$, we can calculate the spectrum via the local data as the limit of spectra ${ }^{7}$

$$
\Gamma\left(X ; \mathcal{Z}_{h}\right)=\lim \left\{\cdots \xi \coprod_{\alpha \beta} \mathcal{H} \mathbb{Z}\left[u, u^{-1}\right] \leftleftarrows \coprod_{\alpha} \mathcal{H} \mathbb{Z}\left[u, u^{-1}\right]\right\},
$$

where again the simplicial homotopy commutative diagram is determined by the transition functions and higher transition data. In practice, this can aid in calculation; however, it is more useful to develop some of the basic properties of the spectrum of sections. Indeed, we will do this in Section 2.2.

We finish our current discussion by defining the underlying twisted cohomology groups for twisted periodic $\mathbb{Z}$-cohomology. Notice that, since the fibers $\mathcal{H} \mathbb{Z}\left[u, u^{-1}\right]$ are 2-periodic, in the sense that $\Sigma^{2} \mathcal{H} \mathbb{Z}\left[u, u^{-1}\right] \simeq \mathcal{H} \mathbb{Z}\left[u, u^{-1}\right]$, and the action by $K(\mathbb{Z}, 2 k)$ commutes with this shift, the sections of $\mathcal{Z}_{h}$ are also 2-periodic.

Definition 2.7 (Twisted periodic integral cohomology). Let $h: X \rightarrow K(\mathbb{Z}, 2 k+1)$ be a twist for periodic integral cohomology. We define the $h$-twisted integral cohomology as the $\mathbb{Z} / 2$-graded group

$$
\widetilde{H}^{*}(X ; h):=\pi_{-*} \Gamma\left(X, \mathcal{Z}_{h}\right) .
$$

We will refer to the degree of a class as either even or odd, corresponding to the identity and nonidentity elements in $\mathbb{Z} / 2$, respectively.

\subsection{Properties of twisted periodic integral cohomology}

In this section, we state some of the basic properties of twisted periodic cohomology, which we generalize to twisted periodic smooth Deligne cohomology in Section 4.2. The following proposition holds more generally for any twisted cohomology theory and is well known (see [62, section 22.1]). We will only state this in the present case for the reduced theory $\widetilde{H}^{*}(X ; h)$ and omit the proof.

Proposition 2.8 (Properties of twisted periodic integral cohomology). Let $X$ be a space and fix a twist as a map $h: X \rightarrow K(\mathbb{Z}, 2 k+1)$. Consider the category of such pairs $(X, h)$, with morphisms $(f, \phi):(X, h) \rightarrow(Y, \ell)$ given by maps $f: X \rightarrow Y$ and equivalences $\phi: f^{*} \ell \Rightarrow h$. The assignment $(X, h) \mapsto \widetilde{H}^{*}(X ; h)$ satisfies the following:

1. $\widetilde{H}^{*}(M ; h)$ is functorial with respect to the maps $f:(X, h) \rightarrow(Y, \ell)$.

2. The functor $\widetilde{H}^{*}(-; h)$ satisfies the Eilenberg-Steenrod axioms for a reduced generalized cohomology theory (i.e., modulo the dimension axiom). In particular,

\footnotetext{
${ }^{7}$ Again, the diagram commutes up to homotopy coherence and the limit is understood in the $\infty$ sense.
} 
we have a Mayer-Vietoris sequence

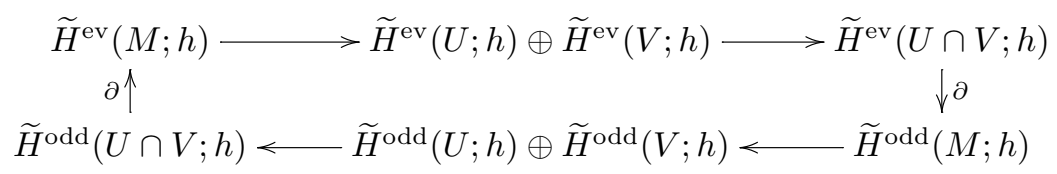

where $\partial$ is the connecting homomorphism, and the sequence is exact at each entry.

3. For $h: X \rightarrow K(\mathbb{Z}, 2 k+1)$ a trivial twist (i.e. $h \simeq *$ ) we have an isomorphism

$$
\widetilde{H}^{*}(X ; h) \cong \widetilde{H}^{*}\left(X ; \mathbb{Z}\left[u, u^{-1}\right]\right) \text {. }
$$

\section{Periodic smooth Deligne cohomology}

In this section, we introduce the notion of periodic Deligne cohomology. This will set the stage for the next section, where we identify the twists of this theory.

\subsection{Construction as a cohomology theory}

Just as the Deligne complex $\mathcal{D}(n)$ is indexed by an integer $n \in \mathbb{Z}$, here we have complexes indexed by elements in $\mathbb{Z} / 2$, which we will call either even or odd, depending on parity. We let $\underline{\mathbb{Z}}$ denote the locally constant sheaf of $\mathbb{Z}$-valued functions.

Definition 3.1 (Even and odd Deligne complexes). For ev, odd $\in \mathbb{Z} / 2$, corresponding to the identity and nonidentity components, respectively, we have the two complexes:

$$
\begin{gathered}
\mathcal{D}(\mathrm{ev}):=(\underbrace{\cdots \longrightarrow \underline{\mathbb{Z}} \oplus \prod_{k} \Omega^{2 k+1} \longrightarrow \prod_{k} \Omega^{2 k} \longrightarrow \underline{\mathbb{Z}} \oplus \prod_{k} \Omega^{2 k+1}}_{\operatorname{deg} \geqslant 0} \longrightarrow \underbrace{0 \longrightarrow \underline{\mathbb{Z}} \longrightarrow \cdots}_{\operatorname{deg}<0}), \\
\mathcal{D}(\text { odd }):=(\underbrace{\cdots \longrightarrow \prod_{k} \Omega^{2 k} \longrightarrow \prod_{k} \Omega^{2 k+1} \longrightarrow \prod_{k} \Omega^{2 k}}_{\operatorname{deg} \geqslant 0} \longrightarrow \underbrace{0 \longrightarrow \underline{\mathbb{Z}} \longrightarrow \cdots}_{\operatorname{deg}<0}),
\end{gathered}
$$

where the $\underline{\mathbb{Z}}$ 's sit in even degrees in the first complex and in odd degrees in the second. In positive degrees, the differential in both complexes is the usual exterior derivative term-wise and on the copies of $\underline{\mathbb{Z}}$ it is given by the inclusion map $\underline{\mathbb{Z}} \hookrightarrow \Omega^{0} \hookrightarrow \prod_{k} \Omega^{2 k}$. In negative degrees the differential is trivial.

The complexes $\mathcal{D}(\mathrm{ev})$ and $\mathcal{D}($ odd $)$ are sheaves of chain complexes on the category of all smooth manifolds, topologized as a site via good open covers. Alternatively, both complexes can be regarded as sheaves of chain complexes on any fixed manifold $M$ simply by evaluating on the open subsets of $M$. This is the familiar setting in which ordinary smooth Deligne cohomology takes place (e.g. [9]). We have the following natural definition.

Definition 3.2 (Periodic Deligne cohomology). We define $\mathbb{Z} / 2$-graded periodic Deligne cohomology groups of a smooth manifold $M$ as the sheaf hypercohomology groups ${ }^{8}$ 
$\widehat{H}^{\mathrm{ev}}\left(M ; \mathbb{Z}\left[u, u^{-1}\right]\right):=H^{0}(M ; \mathcal{D}(\mathrm{ev})) \quad$ and $\quad \widehat{H}^{\text {odd }}\left(M ; \mathbb{Z}\left[u, u^{-1}\right]\right):=H^{0}(M ; \mathcal{D}($ odd $))$.

The following shows that periodic Deligne cohomology can be calculated easily from the ordinary Deligne cohomology groups of a manifold.

Proposition 3.3 (Calculating periodic Deligne cohomology groups). Let $M$ be a smooth manifold. There are natural isomorphisms

$$
\widehat{H}^{\mathrm{ev}}\left(M ; \mathbb{Z}\left[u, u^{-1}\right]\right) \cong \bigoplus_{k} \widehat{H}^{2 k}(M ; \mathbb{Z}) \text { and } \widehat{H}^{\text {odd }}\left(M ; \mathbb{Z}\left[u, u^{-1}\right]\right) \cong \bigoplus_{k} \widehat{H}^{2 k+1}(M ; \mathbb{Z}) \text {. }
$$

Proof. We will prove the claim for $\widehat{H}^{\mathrm{ev}}\left(M ; \mathbb{Z}\left[u, u^{-1}\right]\right)$. The case for $\widehat{H}^{\text {odd }}\left(M ; \mathbb{Z}\left[u, u^{-1}\right]\right)$ is proved similarly. To this end, we organize the sheaf of chain complexes $\mathcal{D}(\mathrm{ev})$ as

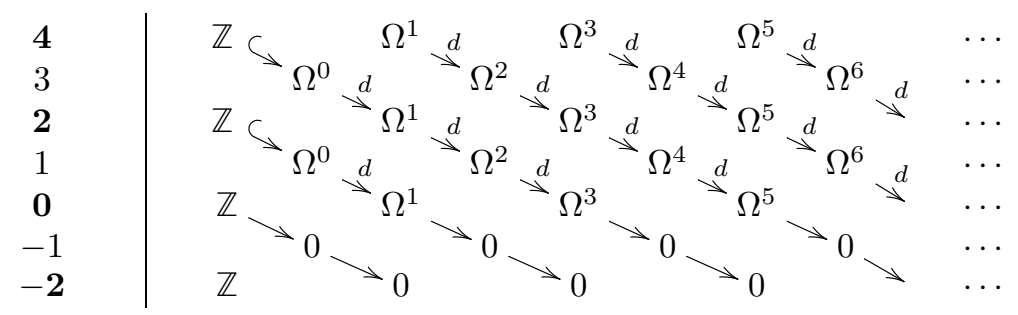

where the numbers on the vertical axis index the degree of the complex. The diagonal arrows represent the differential on each component of the product taken over a given row. The diagonal complexes are easily seen to be the usual Deligne complex and, therefore, we have a splitting

$$
\mathcal{D}(\mathrm{ev}) \cong \prod_{k} \mathcal{D}(2 k) \oplus \prod_{k} \mathbb{Z}[-2 k],
$$

where the second summand comes from the negative degrees of the complex. The latter do not contribute to the hypercohomology of the complex, as the Čech resolution of the complex necessarily vanishes in negative degrees. Thus, the hypercohomology groups split as desired.

From Proposition 3.3, it follows immediately that the periodic Deligne cohomology groups fit into a differential cohomology diamond diagram and into exact sequences similar to those for ordinary Deligne cohomology, as an instance of differential integral cohomology [75].

Proposition 3.4 (Periodic Deligne cohomology diamond). We have an exact diagram

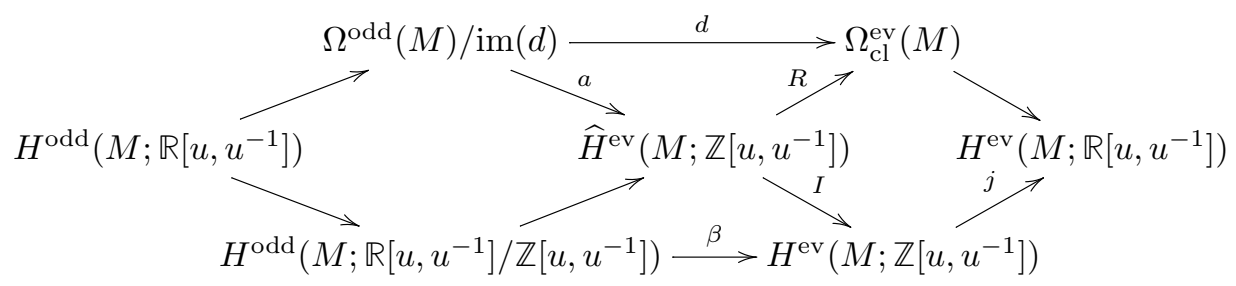

for the even Deligne complex and a similar diamond for the odd one, given by

\footnotetext{
${ }^{8}$ The cohomological degrees on both right hand sides is 0 , due to the shift in the complexes in Definition 3.1, in analogy to the usual Deligne case, i.e., expression (1.1).
} 
switching ev and odd. Here $\Omega^{\mathrm{odd}}(M)$ and $\Omega^{\mathrm{ev}}(M)$ are the groups of differential forms of odd and even degrees, respectively. For example, an element $\omega \in \Omega^{\mathrm{ev}}(M)$ is a formal combination $\omega=\omega_{0}+\omega_{2}+\omega_{4}+\cdots$, with $\omega_{2 i}$ a differential form of degree $2 i$.

Remark 3.5 (Extension of the diamond to a long exact sequence). One of the diagonals in the diamond diagram in Proposition 3.4 can be extended to a long exact sequence. Depending on parity, the relevant segments of this long exact sequence are

$$
\begin{aligned}
& H^{\mathrm{ev}}\left(M ; \mathbb{Z}\left[u, u^{-1}\right]\right) \rightarrow \Omega^{\mathrm{ev}}(M) / \operatorname{im}(d) \rightarrow \widehat{H}^{\mathrm{odd}}\left(M ; \mathbb{Z}\left[u, u^{-1}\right]\right) \rightarrow H^{\text {odd }}\left(M ; \mathbb{Z}\left[u, u^{-1}\right]\right) \rightarrow 0, \\
& H^{\text {odd }}\left(M ; \mathbb{Z}\left[u, u^{-1}\right]\right) \rightarrow \Omega^{\text {odd }}(M) / \operatorname{im}(d) \rightarrow \widehat{H}^{\mathrm{ev}}\left(M ; \mathbb{Z}\left[u, u^{-1}\right]\right) \rightarrow H^{\mathrm{ev}}\left(M ; \mathbb{Z}\left[u, u^{-1}\right]\right) \rightarrow 0 .
\end{aligned}
$$

The map into the quotient $\Omega^{\mathrm{ev}}(M) / \operatorname{im}(d)$ takes a periodic integral class and maps it to the class of its corresponding de Rham representative (i.e. a form with integral periods). Note also that the map $R: \widehat{H}^{\mathrm{ev}}\left(M ; \mathbb{Z}\left[u, u^{-1}\right]\right) \rightarrow \Omega_{\mathrm{cl}}^{\mathrm{ev}}(M)$ is not surjective; its image is the subgroup of closed forms with integral periods.

\section{2. $\quad$ Ring structure and examples}

Eventually, we would like to consider the twists of this theory and, to do this, we need a ring structure on this periodic Deligne complex. Recall that for ordinary Deligne cohomology, the Deligne-Beilinson cup product gives a collection of morphisms of sheaves of chain complexes $[22,6]$ (see also $[25,27]$ )

$$
\cup_{\mathrm{DB}}: \mathcal{D}(n) \otimes \mathcal{D}(m) \longrightarrow \mathcal{D}(n+m) \text {. }
$$

At the level of local sections, it is defined by the formula

$$
\alpha \cup_{\mathrm{DB}} \beta=\left\{\begin{array}{cl}
\alpha \beta, & \operatorname{deg}(\alpha)=n, \\
\alpha \wedge d \beta, & \operatorname{deg}(\beta)=0, \operatorname{deg}(\alpha) \neq n, \\
0, & \text { otherwise }
\end{array}\right.
$$

Since the even periodic Deligne complex split as the product (3.1) (and similarly for the odd), there are multiplication maps

$$
\cup_{\mathrm{DB}}:\left\{\begin{array}{l}
\mathcal{D}(\text { ev }) \otimes \mathcal{D}(\text { ev }) \longrightarrow \mathcal{D}(\text { ev }) \\
\mathcal{D}(\text { ev }) \otimes \mathcal{D}(\text { odd }) \longrightarrow \mathcal{D}(\text { odd }) \\
\mathcal{D}(\text { odd }) \otimes \mathcal{D}(\text { odd }) \longrightarrow \mathcal{D}(\text { ev })
\end{array}\right.
$$

induced by the cup product $\cup_{\mathrm{DB}}$ from (3.2) in positive degrees and the multiplication of integers in negative degrees. It is immediate that these maps descend to a graded commutative cup product which is compatible with the Deligne-Beilinson cup product term-wise. We summarize these observations as follows.

Proposition 3.6 (Superalgebra structure on periodic Deligne cohomology). With the multiplication maps (3.3) induced by the Deligne-Beilinson cup product, the complex $\mathcal{D}(\mathrm{ev}) \oplus \mathcal{D}$ (odd) admits the structure of a sheaf of differentially graded superalgebras. At the level of hypercohomology, it gives

$$
\widehat{H}^{\mathrm{ev} / \text { odd }}\left(M ; \mathbb{Z}\left[u, u^{-1}\right]\right):=\widehat{H}^{\mathrm{ev}}\left(M ; \mathbb{Z}\left[u, u^{-1}\right]\right) \oplus \widehat{H}^{\mathrm{odd}}\left(M ; \mathbb{Z}\left[u, u^{-1}\right]\right)
$$


the structure of a commutative superalgebra. Moreover, we have

$$
\begin{gathered}
\widehat{H}^{\mathrm{ev} / \text { odd }}\left(M ; \mathbb{Z}\left[u, u^{-1}\right]\right) \otimes \widehat{H}^{\mathrm{ev} / \text { odd }}\left(M ; \mathbb{Z}\left[u, u^{-1}\right]\right) \stackrel{\cup_{\mathrm{DB}}}{\longrightarrow} \widehat{H}^{\mathrm{ev} / \text { odd }}\left(M ; \mathbb{Z}\left[u, u^{-1}\right]\right) \\
\downarrow \\
H^{\mathrm{ev} / \text { odd }}\left(M ; \mathbb{Z}\left[u, u^{-1}\right]\right) \otimes H^{\mathrm{ev} / \text { odd }}\left(M ; \mathbb{Z}\left[u, u^{-1}\right]\right) \stackrel{\cup}{\longrightarrow} H^{\mathrm{ev} / \text { odd }}\left(M ; \mathbb{Z}\left[u, u^{-1}\right]\right), \\
\widehat{H}^{\mathrm{ev} / \text { odd }}\left(M ; \mathbb{Z}\left[u, u^{-1}\right]\right) \otimes \widehat{H}^{\mathrm{ev} / \text { odd }}\left(M ; \mathbb{Z}\left[u, u^{-1}\right]\right) \stackrel{\cup_{\mathrm{DB}}}{\longrightarrow} \widehat{H}^{\mathrm{ev} / \text { odd }}\left(M ; \mathbb{Z}\left[u, u^{-1}\right]\right) \\
\downarrow \\
\Omega_{\mathrm{cl}}^{\mathrm{ev} / \text { odd }}(M) \otimes \Omega_{\mathrm{cl}}^{\mathrm{ev} / \text { odd }}(M) \longrightarrow \Omega_{\mathrm{cl}}^{\mathrm{ev} / \text { odd }}(M)
\end{gathered}
$$

where $H^{\mathrm{ev} / \text { odd }}\left(M ; \mathbb{Z}\left[u, u^{-1}\right]\right)$ is periodic integral cohomology, endowed with the superalgebra structure inherited from the cup product, and $\Omega^{\mathrm{ev} / \mathrm{odd}}(M)$ is the superalgebra of graded differential forms.

Example 3.7 (Periodic Deligne cohomology of even spheres). The underlying periodic integral cohomology of the smooth $2 k$-sphere is readily computed as

$$
H^{\mathrm{ev}}\left(S^{2 k} ; \mathbb{Z}\left[u, u^{-1}\right]\right) \cong \mathbb{Z} \oplus \mathbb{Z} \quad \text { and } \quad H^{\text {odd }}\left(S^{2 k} ; \mathbb{Z}\left[u, u^{-1}\right]\right) \cong 0 .
$$

Given the two long exact sequences in Remark 3.5, we easily compute

$$
\begin{aligned}
\widehat{H}^{\mathrm{ev}}\left(S^{2 k} ; \mathbb{Z}\left[u, u^{-1}\right]\right) & \cong \Omega^{\mathrm{odd}}\left(S^{2 k}\right) / \operatorname{im}(d) \oplus \mathbb{Z} \oplus \mathbb{Z}, \\
\widehat{H}^{\mathrm{odd}}\left(S^{2 k} ; \mathbb{Z}\left[u, u^{-1}\right]\right) & \cong \Omega^{\mathrm{ev}}\left(S^{2 k}\right) / \Omega_{\mathrm{cl}, \mathbb{Z}}^{\mathrm{ev}}\left(S^{2 k}\right),
\end{aligned}
$$

where $\Omega_{\mathrm{cl}, \mathbb{Z}}^{\mathrm{ev}}\left(S^{2 k}\right)$ is the subgroup of closed even forms with integral periods (i.e., each component of an element $\omega=\omega_{0}+\omega_{2}+\cdots$ has integral periods).

Example 3.8 (Periodic Deligne cohomology of odd spheres). Similarly, we calculate for odd spheres using the same two sequences above, to get in this case

$$
\begin{aligned}
& \widehat{H}^{\text {odd }}\left(S^{2 k+1} ; \mathbb{Z}\left[u, u^{-1}\right]\right) \cong \Omega^{\mathrm{ev}}\left(S^{2 k+1}\right) / \operatorname{im}(d) \oplus \mathbb{Z}, \\
& \widehat{H}^{\mathrm{ev}}\left(S^{2 k+1} ; \mathbb{Z}\left[u, u^{-1}\right]\right) \cong \Omega^{\text {odd }}\left(S^{2 k+1}\right) / \Omega_{\mathrm{cl}, \mathbb{Z}}^{\text {odd }}\left(S^{2 k+1}\right) \oplus \mathbb{Z},
\end{aligned}
$$

where one of the $\mathbb{Z}$ factors has moved, in comparison to the case of even spheres, due to parity reasons.

\section{Twisted periodic smooth Deligne cohomology}

In this section we turn to twisting periodic Deligne cohomology constructed above. Just as twisted periodic integral cohomology in Section 2.1 takes the form of a bundle of spectra over a parametrizing space, here we will have a smooth bundle of spectra, parametrized over a smooth manifold $M$. In the smooth setting, our starting point is no longer the category of spaces and its tangent infinity category $T$ (Space), but rather the category of smooth stacks $\mathcal{S} h_{\infty}(\mathcal{M} f)$ and its tangent infinity category $T\left(\mathcal{S h} \mathrm{L}_{\infty}(\mathcal{M} \mathrm{f})\right)$.

\subsection{The parametrized spectrum and gerbes via smooth stacks}

Let $M$ be a smooth manifold and consider the site of open subsets $\mathcal{O}$ pen $(M)$, topologized via the good open covers $\left\{U_{\alpha} \rightarrow M\right\}$. Smooth stacks on $M$ are similar to 
smooth sheaves, but instead of assigning a set of elements to an object $U \in \mathcal{O} \operatorname{pen}(M)$, we assign a space (usually modeled combinatorially by a simplicial set). The sheaf gluing condition is replaced by a weaker condition, where we only require gluing up to equivalence.

There is a large $\infty$-category of smooth stacks $\mathcal{S} \mathrm{h}_{\infty}(\mathcal{M f})$ which does not depend on a choice of underlying smooth manifold. The site for this $\infty$-category is the site of all smooth manifolds $\mathcal{M} f$, topologized via good open covers. Any object in $\mathcal{S h}_{\infty}(\mathcal{M f})$ can be restricted to a single manifold by simply considering its value on open subsets $U \hookrightarrow M$. One of the benefits of working in this larger $\infty$-category is that one can define moduli stacks $\mathbf{X}$ which represent objects of interest over $M$ via maps $M \rightarrow \mathbf{X}$. For example, the moduli stack of higher gerbes with connection $\mathbf{B}^{n} U(1)_{\nabla}$ was studied in $[29,71,74,27,28]$. One way to present this stack is by applying the Dold-Kan functor to the sheaf of chain complexes

$$
\mathbf{B}^{n} U(1)_{\nabla}=\operatorname{DK}\left(\cdots \longrightarrow 0 \longrightarrow \underline{U(1)} \stackrel{d \log }{\longrightarrow} \Omega^{1} \stackrel{d}{\longrightarrow} \Omega^{2} \stackrel{d}{\longrightarrow} \cdots \longrightarrow \Omega^{n}\right),
$$

where the sheaf $U(1):=C^{\infty}(-; U(1))$ sits in degree $n$. The sheaf in the argument of DK is quasi-isomorphic (via the exponential map) to the smooth Deligne complex $\mathcal{D}(n)$. The Dold-Kan functor sends quasi-isomorphisms to weak equivalences and (since we are working up to equivalence) this justifies the uniform notation $\mathbf{B}^{n} U(1)_{\nabla}$ for both resulting stacks (i.e. upon applying DK to either complex). The stack $\mathbf{B}^{n} U(1)_{\nabla}$ sits in a Cartesian square

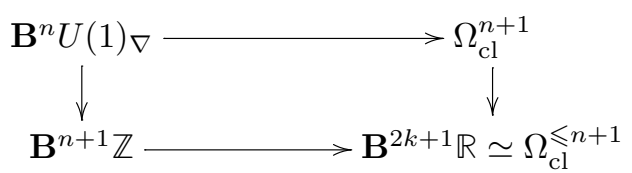

where $\Omega_{\mathrm{cl}}^{\leqslant n+1}$ is the stack presented by the sheaf of chain complexes

$$
\left(\cdots \longrightarrow 0 \longrightarrow \Omega^{0} \longrightarrow \Omega^{1} \stackrel{d}{\longrightarrow} \Omega^{2} \stackrel{d}{\longrightarrow} \cdots \longrightarrow \Omega_{\mathrm{cl}}^{n+1}\right) .
$$

In [29], it was shown that the homotopy classes of maps $M \rightarrow \mathbf{B}^{n} U(1)_{\nabla}$ is in bijective correspondence with the Deligne cohomology group $\widehat{H}^{n}(M ; \mathbb{Z})$.

Example 4.1 (Stack of 2-bundles with connections/gerbes with connections). The smooth stack $\mathbf{B}^{2} U(1)_{\nabla}$ can be presented via the Dold-Kan correspondence by the sheaf of positively graded chain complexes

$$
\mathbf{B}^{2} U(1)_{\nabla}=L \circ \mathrm{DK}\left(\underline{U(1)} \stackrel{d \log }{\longrightarrow} \Omega^{1} \stackrel{d}{\longrightarrow} \Omega^{2}\right),
$$

where $L$ is the stackification functor. ${ }^{9}$ Let $\phi: \mathbb{R}^{n} \rightarrow M$ be a local chart. For a convex open subset $U \subset \mathbb{R}^{n}$, this stack can be evaluated on the corresponding open subset $V=\phi(U)$ via

$$
\operatorname{Map}\left(V, \mathbf{B}^{2} U(1)_{\nabla}\right) \stackrel{\phi}{\simeq} \mathrm{DK}\left(C^{\infty}(U, U(1)) \stackrel{d \log }{\longrightarrow} \Omega^{1}(U) \stackrel{d}{\longrightarrow} \Omega^{2}(U)\right) .
$$

More generally, descent for the stack $\mathbf{B}^{2} U(1)_{\nabla}$ implies that, for any choice of good

\footnotetext{
${ }^{9}$ This is a functor which turns a prestack into a stack, analogously to the way a sheafification functor turns a presheaf into a sheaf. See [56, Sec. 6.5.3] for details.
} 
open cover $\left\{U_{\alpha}\right\}$ of $M$, the space of maps $\operatorname{Map}\left(M, \mathbf{B}^{2} U(1)_{\nabla}\right)$ can be identified by replacing $M$ with the Čech nerve $\check{C}\left(\left\{U_{\alpha}\right\}\right)$ of $\left\{U_{\alpha}\right\}$ and considering instead the space

$$
\operatorname{Map}\left(\check{C}\left(\left\{U_{\alpha}\right\}\right), \operatorname{DK}\left(\underline{U(1)} \stackrel{d \log }{\longrightarrow} \Omega^{1} \stackrel{d}{\longrightarrow} \Omega^{2}\right)\right) .
$$

By the basic properties of the Dold-Kan correspondence we have an isomorphism ${ }^{10}$

$$
\pi_{0}\left(\operatorname{Map}\left(\check{C}\left(\left\{U_{\alpha}\right\}\right), \operatorname{DK}\left(\underline{U(1)} \stackrel{d \log }{\longrightarrow} \Omega^{1} \stackrel{d}{\longrightarrow} \Omega^{2}\right)\right)\right) \cong H^{2}\left(M ; \underline{U(1)} \stackrel{d \log }{\longrightarrow} \Omega^{1} \stackrel{d}{\longrightarrow} \Omega^{2}\right) .
$$

By [9, Theorem 5.3.11], the elements on the right parametrize the homotopy classes of the gerbes with connective structure and curving.

Definition 4.2 (Smooth parametrized spectrum). A smooth parametrized prespectrum is a collection of morphisms $p_{n}: E_{n} \rightarrow M$ between smooth stacks in $\mathcal{S h}_{\infty}(\mathcal{M f f})$, $n \in \mathbb{Z}$, with a choice of section, equipped with morphisms $\Sigma_{M} E_{n} \rightarrow E_{n+1}$, making certain diagrams commute up to a choice of equivalence in $\mathcal{S} h_{\infty}(\mathcal{M f})$. A smooth parametrized prespectrum $\left\{p_{n}: E_{n} \rightarrow M\right\}$ for which the adjoint maps $E_{n} \rightarrow \Omega_{M} E_{n+1}$ are equivalences is called smooth parametrized spectrum.

Remark 4.3 (Identifying the proper category as a setting). Note that Definition 4.2 is almost verbatim the same as one has for parametrized spectra, the only difference being where the objects $E_{n}$ and $M$ live (i.e. smooth stacks instead of spaces). In this context we still have a mapping spectrum ${ }^{11}$ between two smooth spectra. The resulting structure is again an $\infty$-category and we denote this category by $T\left(\mathcal{S h}_{\infty}(\mathcal{M} f)\right)$.

Definition 4.4 (Smooth bundle of spectra). Let $M$ be a smooth manifold. A smooth bundle of spectra $\pi: E \rightarrow M$ over $M$ with fiber the sheaf of spectra $\mathcal{R}$ is an object in $T\left(\mathcal{S h} \mathrm{h}_{\infty}(\mathcal{M f})\right)_{M}$ satisfying the same properties of the underlying topological bundles of spectra, with $M$ replacing $X$.

We now wish to focus our scope to the case of periodic Deligne cohomology. Consider the sheaf of ring spectra given by applying the Eilenberg-MacLane functor $\mathcal{H}$ to the sheaf of chain complexes $\mathcal{D}(\mathrm{ev})$ and $\mathcal{D}$ (odd). In Section 3.1, we saw that this ring spectrum represents periodic Deligne cohomology, in the sense that

$$
\begin{aligned}
\widehat{H}^{\mathrm{ev}}\left(M ; \mathbb{Z}\left[u, u^{-1}\right]\right) & \cong \pi_{0} \operatorname{Map}(M ; \mathcal{H}(\mathcal{D}(\text { ev }))), \\
\widehat{H}^{\text {odd }}\left(M ; \mathbb{Z}\left[u, u^{-1}\right]\right) & \cong \pi_{0} \operatorname{Map}(M ; \mathcal{H}(\mathcal{D}(\text { odd }))) .
\end{aligned}
$$

We would like to identify a large class of twists for this theory. To this end, let us consider the stack of twists in diagram (1.2) with $\widehat{\mathcal{R}}$ the periodic differential ring spectrum given by both $\mathcal{H}(\mathcal{D}(\mathrm{ev}))$ and $\mathcal{H}(\mathcal{D}(\mathrm{odd}))$, separately. At first, it might appear that we get two stacks of twists corresponding to both the even and odd degrees; however, this is not the case.

\footnotetext{
${ }^{10}$ The shift in degree occurs because on the left we consider the complex $U(1) \stackrel{d \log }{\longrightarrow} \Omega^{1} \stackrel{d}{\longrightarrow} \Omega^{2}$ as being shifted up two degrees relative to the complex appearing on the right.

${ }^{11}$ Note that the mapping spectra are not smooth or parametrized; they are ordinary topological spectra.
} 
Proposition 4.5 (Equivalence of stacks of even and odd twists for periodic Deligne cohomology). We have a canonical equivalence of smooth stacks

$$
\widehat{\mathrm{Tw}}_{\mathcal{H}(\mathcal{D}(\text { odd }))} \simeq \widehat{\mathrm{Tw}}_{\mathcal{H}(\mathcal{D}(\mathrm{ev}))}
$$

induced by shifting both the ring spectrum $\mathcal{H}\left(\mathbb{Z}\left[u, u^{-1}\right]\right)$ and the invertible periodic de Rham complex $\Omega^{*}\left[u, u^{-1}\right]$ up by one degree each.

Proof. Shifting a module spectrum $\mathcal{R}_{\tau}$ up by one degree is again a module spectrum over the ring spectrum $\mathcal{R}$, i.e., the module maps $\mu: \mathcal{R}^{m} \wedge \mathcal{R}_{h}^{n} \rightarrow \mathcal{R}_{h}^{m+n}$ give rise to maps $\mathcal{R}^{m} \wedge \mathcal{R}_{h}^{n+1} \rightarrow \mathcal{R}_{h}^{m+n+1}$, and the higher coherence homotopies in the ring structure give rise to coherence homotopies by shifting. Similarly, shifting a Kflat invertible module $\mathcal{L}$ is again a $\mathrm{K}$-flat invertible module. Moreover, given any equivalence $\mathcal{H}(\mathcal{L}) \simeq \mathcal{R}_{\tau} \wedge \mathcal{H} \mathbb{R}$, we get a corresponding equivalence at the level of the shifts. By the universal property of the pullback, we have an induced map at the level of the twists. For smooth periodic Deligne cohomology this takes the form $\widehat{\mathrm{Tw}}_{\mathcal{H}(\mathcal{D}(\text { odd }))} \rightarrow \widehat{\mathrm{Tw}}_{\mathcal{H}(\mathcal{D}(\mathrm{ev}))}$. It is immediate that this map admits an inverse induced by shifting down.

Proposition 4.5 implies that we do not have to consider the even and odd degrees separately, but we can view a given twist as corresponding to either spectrum. Henceforth, we will only refer to the stack of twists of periodic Deligne cohomology and denote it by $\widehat{\mathrm{Tw}}$.

The main goal of this section is to show that periodic Deligne cohomology can be twisted by odd degree gerbes with connection, i.e., we want to construct a map (or representation)

$$
\rho: \mathbf{B}^{2 n+1} U(1)_{\nabla} \longrightarrow \widehat{\mathrm{TW}} .
$$

Although the existence of such twists follows from [15, proposition 11.4], the argument used there is rather abstract, and does not reveal precisely how the topological and geometric twists combine to form a twist of the differential theory. For this reason, we present an alternative proof which makes these relationships more manifest.

We now proceed with the construction, in which the above representation is essentially provided by Chern-Simons type data associated to a Čech de Rham cocycle. Consider a Čech de Rham cochain $\eta=\left(\eta_{\alpha}, \eta_{\alpha_{0} \alpha_{1}}, \ldots, \eta_{\alpha_{0} \alpha_{1} \cdots \alpha_{2 k+1}}\right)$ on a smooth manifold $M$, where $\eta_{\alpha_{0} \cdots \alpha_{i}}$ is the cocycle data on the $i$-fold intersections of a fixed good open cover $\left\{U_{\alpha}\right\}$, i.e., $\eta_{\alpha}$ is a $2 k$-form defined on open sets, $\eta_{\alpha_{0} \alpha_{1}}$ is a $(2 k-1)$ form defined on intersections, etc. We can regard this cochain as an element in degree 1 of the total complex $\operatorname{Tot}^{\bullet}\left(\left\{U_{\alpha}\right\} ; \Omega^{\leqslant 2 k+1}\right)$, where

$$
\Omega^{\leqslant 2 k+1}:=\left(\cdots \longrightarrow 0 \longrightarrow \Omega^{0} \longrightarrow \Omega^{1} \longrightarrow \cdots \longrightarrow \Omega_{\mathrm{cl}}^{2 k+1}\right),
$$

with $\Omega^{0}$ in degree $(2 k+1)$. Choose this cochain such that $D(\eta)=H-\left(h_{\alpha_{0} \cdots \alpha_{2 k+1}}\right)$ with $D=d+(-1)^{i} \delta$ the total differential, $H$ a closed globally defined $(2 k+1)$-form and $\left(h_{\alpha_{0} \cdots \alpha_{2 k+1}}\right)$ a real valued Čech cocycle.

To the above data we will associate an equivalence of sheaves of spectra

$$
\mathrm{CS}(\eta) \wedge(-): \mathcal{H}\left(\mathbb{R}\left[u, u^{-1}\right]\right)_{h}(M) \stackrel{\simeq}{\longrightarrow} \mathcal{H}\left(\Omega^{*}\left[u, u^{-1}\right], d_{H}\right)(M),
$$

where $\left(\Omega^{*}\left[u, u^{-1}\right], d_{H}\right)(M)$ is the $H$-twisted de-Rham complex. Consider the local 
forms

$$
\begin{aligned}
\mathrm{CS}_{\alpha}(\eta) & =e^{\eta_{\alpha}}=1+\eta_{\alpha}+\frac{1}{2 !} \eta_{\alpha} \wedge \eta_{\alpha}+\frac{1}{3 !} \eta_{\alpha} \wedge \eta_{\alpha} \wedge \eta_{\alpha}+\cdots \\
\mathrm{CS}_{\alpha_{0} \alpha_{1}}(\eta) & =\eta_{\alpha_{0} \alpha_{1}}+\frac{1}{2 !} \eta_{\alpha_{0} \alpha_{1}} \wedge d \eta_{\alpha_{0} \alpha_{1}}+\frac{1}{3 !} \eta_{\alpha_{0} \alpha_{1}} \wedge d \eta_{\alpha_{0} \alpha_{1}} \wedge \eta_{\alpha_{0} \alpha_{1}}+\cdots \\
\mathrm{CS}_{\alpha_{0} \alpha_{1} \alpha_{2}}(\eta) & =\eta_{\alpha_{0} \alpha_{1} \alpha_{2}}+\frac{1}{2 !} \eta_{\alpha_{0} \alpha_{1} \alpha_{2}} \wedge d \eta_{\alpha_{0} \alpha_{1}}+\frac{1}{3 !} \eta_{\alpha_{0} \alpha_{1} \alpha_{2}} \wedge d \eta_{\alpha_{0} \alpha_{1}} \wedge d \eta_{\alpha_{0} \alpha_{1}}+\cdots
\end{aligned}
$$

and so on, with $\mathrm{CS}_{\alpha_{0} \alpha_{1} \cdots \alpha_{i}}$ being defined on the $i$-fold intersection.

Lemma 4.6 (CS inducing a morphism). For a periodic form $\omega$ in $\left.\Omega^{*}\left[u, u^{-1}\right]\right|_{U_{\alpha \beta}}$, the assignment $\omega \mapsto \mathrm{CS}_{\alpha}(\eta) \wedge \omega$ defines a morphism of restricted sheaves of complexes

$$
\mathrm{CS}_{\alpha}(\eta) \wedge(-):\left.\left.\left(\Omega^{*}\left[u, u^{-1}\right], d_{H}\right)\right|_{U_{\alpha}} \longrightarrow \Omega^{*}\left[u, u^{-1}\right]\right|_{U_{\alpha}},
$$

which induces a morphism of sheaves of spectra

$$
\mathcal{H}\left(\mathrm{CS}_{\alpha}(\eta) \wedge(-)\right):\left.\left.\mathcal{H}\left(\Omega^{*}\left[u, u^{-1}\right], d_{H}\right)\right|_{U_{\alpha}} \longrightarrow \mathcal{H}\left(\Omega^{*}\left[u, u^{-1}\right]\right)\right|_{U_{\alpha}} .
$$

Proof. This follows immediately from the calculation

$$
d\left(e^{\eta_{\alpha}} \wedge \omega\right)=H \wedge e^{\eta_{\alpha}} \wedge \omega+e^{\eta_{\alpha}} \wedge d \omega=e^{\eta_{\alpha}} \wedge(H \wedge \omega+d \omega)=e^{\eta_{\alpha}} \wedge d_{H}(\omega)
$$

and that $\mathcal{H}$ is functorial.

We can think of (4.2) as the map on local sections induced by a local trivialization of the bundle of spectra corresponding to the module spectrum $\mathcal{H}\left(\Omega^{*}\left[u, u^{-1}\right], d_{H}\right)$. Since $d \eta_{\alpha_{0} \alpha_{1}}=\delta \eta_{\alpha}$, by construction, it follows that $d \mathrm{CS}_{\alpha_{0} \alpha_{1}}(\eta)=e^{d \eta_{\alpha_{0} \alpha_{1}}}=e^{\delta \eta_{\alpha}}=$ $\delta \mathrm{CS}_{\alpha}(\eta)$, where $\delta \mathrm{CS}_{\alpha}(\eta)$ should be understood as the alternating wedge product.

Lemma 4.7 (CS defining a chain homotopy). The assignment $\omega \mapsto \mathrm{CS}_{\alpha_{0} \alpha_{1}}(\eta) \wedge \omega$ defines a chain homotopy trivializing the degree zero element

$$
\delta \mathrm{CS}_{\alpha}(\eta) \wedge(-) \in \operatorname{hom}\left(\left.\Omega^{*}\left[u, u^{-1}\right]\right|_{U_{\alpha \beta}},\left.\Omega^{*}\left[u, u^{-1}\right]\right|_{U_{\alpha \beta}}\right) .
$$

Proof. For $X$ and $Y$ two complexes, the differential on $\operatorname{hom}(X, Y)$ acts on an element $f_{n}$ in degree $n$ by $d_{Y} f_{n}(x)-(-1)^{n} f_{n}\left(d_{X} x\right)$. For $X=Y=\left.\Omega^{*}\left[u, u^{-1}\right]\right|_{U_{\alpha \beta}}$, the assignment $\omega \mapsto \mathrm{CS}^{(1)} \wedge \omega$ manifestly gives an element in degree 1 and the differential thus acts by

$$
d\left(\mathrm{CS}_{\alpha_{0} \alpha_{1}}(\eta) \wedge \omega\right)+\mathrm{CS}_{\alpha_{0} \alpha_{1}}(\eta) \wedge d \omega=d\left(\mathrm{CS}_{\alpha}(\eta)\right) \wedge \omega=\delta \mathrm{CS}_{\alpha}(\eta) \wedge \omega .
$$

The Eilenberg-MacLane functor is a fully faithful $\infty$-functor and we have a natural equivalence of mapping spaces

$$
\begin{aligned}
\operatorname{Map}\left(\left.\Omega^{*}\left[u, u^{-1}\right]\right|_{U_{\alpha \beta}},\left.\Omega^{*}\left[u, u^{-1}\right]\right|_{U_{\alpha \beta}}\right) & :=\Omega^{\infty} \mathcal{H}\left(\operatorname{hom}\left(\left.\Omega^{*}\left[u, u^{-1}\right]\right|_{U_{\alpha \beta}},\left.\Omega^{*}\left[u, u^{-1}\right]\right|_{U_{\alpha \beta}}\right)\right) \\
& \simeq \operatorname{Map}\left(\mathcal{H}\left(\left.\Omega^{*}\left[u, u^{-1}\right]\right|_{U_{\alpha \beta}}\right), \mathcal{H}\left(\left.\Omega^{*}\left[u, u^{-1}\right]\right|_{U_{\alpha \beta}}\right)\right) .
\end{aligned}
$$

Hence, in particular, chain homotopies are mapped to homotopies between sheaves of spectra. From Lemma 4.7, it immediately follows that $\mathrm{CS}_{\alpha_{0} \alpha_{1}}(\eta)$ gives rise to a 
homotopy filling the diagram

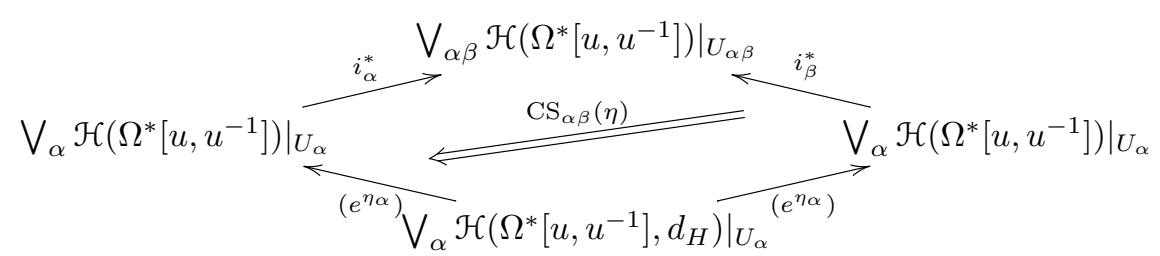

Lemma 4.8 (Čech differential of CS). The assignment $\omega \mapsto \mathrm{CS}_{\alpha_{0} \cdots \alpha_{i}}(\eta) \wedge \omega$ gives a degree $0<i \leqslant 2 k$ element

$$
\mathrm{CS}_{\alpha_{0} \cdots \alpha_{i}}(\eta) \wedge(-) \in \operatorname{hom}^{i}\left(\left.\Omega^{*}\left[u, u^{-1}\right]\right|_{U_{\alpha_{0} \cdots \alpha_{i}}},\left.\Omega^{*}\left[u, u^{-1}\right]\right|_{U_{\alpha_{0} \cdots \alpha_{i}}}\right)
$$

trivializing $\delta \operatorname{CS}_{\alpha_{0} \cdots \alpha_{i-1}}(\eta) \wedge(-)$. Moreover, in degree $2 k$, we have

$$
\begin{aligned}
\delta \mathrm{CS}_{\alpha_{0} \cdots \alpha_{2 k}}(\eta)= & h_{\alpha_{0} \alpha_{1} \cdots \alpha_{2 k+1}}+\frac{1}{2 !} h_{\alpha_{0} \alpha_{1} \cdots \alpha_{2 k+1}} \wedge d \eta_{\alpha_{0} \alpha_{1}}+ \\
& +\frac{1}{3 !} h_{\alpha_{0} \alpha_{1} \cdots \alpha_{2 k+1}} \wedge d \eta_{\alpha_{0} \alpha_{1}} \wedge d \eta_{\alpha_{0} \alpha_{1}}+\cdots \\
=h_{\alpha_{0} \cdots \alpha_{2 k+1}} & \operatorname{CS}^{\prime}(\eta)
\end{aligned}
$$

where $\mathrm{CS}_{\alpha}^{\prime}(\eta):=1+\frac{1}{2 !} d \eta_{\alpha}+\frac{1}{3 !} d \eta_{\alpha} \wedge d \eta_{\alpha} \cdots$.

Proof. Again, by construction we have $d \mathrm{CS}_{\alpha_{0} \cdots \alpha_{i}}(\eta)=(-1)^{i+1} \delta\left(\mathrm{CS}_{\alpha_{0} \cdots \alpha_{i-1}}(\eta)\right)$. The same computation as in Lemma 4.7 with $n=i$ shows that $\operatorname{CS}_{\alpha_{0} \cdots \alpha_{i}}(\eta) \wedge(-)$ indeed trivializes $\mathrm{CS}_{\alpha_{0} \cdots \alpha_{i-1}}(\eta) \wedge(-)$. The second claim follows immediately from the fact that $\eta$ was chosen so that $\delta\left(\eta_{\alpha_{0} \cdots \alpha_{2 k}}\right)=h_{\alpha_{0} \cdots \alpha_{2 k+1}}$.

The combinatorial data provided by the cochain $\operatorname{CS}(\eta)$ is precisely what is needed to specify an $\infty$-cone over the cosimplicial diagram

$$
\left.\left.\cdots \xi \bigvee_{\alpha \beta} \mathcal{H}\left(\Omega^{*}\left[u, u^{-1}\right]\right)\right|_{U_{\alpha \beta}} \sum_{i_{\alpha}^{*}}^{i_{\beta}^{*}} \bigvee_{\alpha} \mathcal{H}\left(\Omega^{*}\left[u, u^{-1}\right]\right)\right|_{U_{\alpha}} \stackrel{\mathrm{CS}_{\alpha}(\eta)}{\gtrless} \mathcal{H}\left(\Omega^{*}\left[u, u^{-1}\right], d_{H}\right),
$$

where all homotopies in the cosimplicial diagram are degenerate except at the $(2 k+$ 1)-stage, where we fill the diagram by a nontrivial homotopy determined by the equation $\delta i_{\alpha_{0} \cdots \alpha_{2 k}}^{*}=h_{\alpha_{0} \cdots \alpha_{2 k+1}} \cup(-)$. This is equivalent to specifying a map

$$
h_{\alpha_{0} \cdots \alpha_{2 k+1}}: \Delta^{2 k+1} \longrightarrow \operatorname{Map}\left(\left.\mathcal{H}\left(\Omega^{*}\left[u, u^{-1}\right]\right)\right|_{U_{\alpha}},\left.\mathcal{H}\left(\Omega^{*}\left[u, u^{-1}\right]\right)\right|_{U_{\alpha_{0} \cdots \alpha_{2 k+1}}}\right),
$$

where the top face acts as a higher homotopy via the cup product and the restriction to the faces are degenerate on the restriction to $(2 k+1)$-fold intersections.

Example 4.9. Note that the homotopy commutative diagram (4.3) gives homotopy commutativity at the first stage. We can organize the homotopies at the next stage diagrammatically as

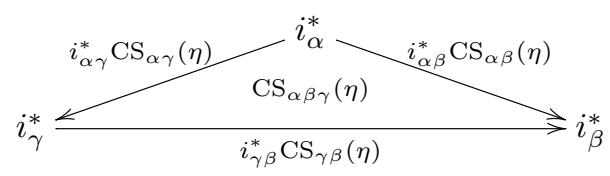

The only nontrivial step is on the $(2 k+1)$-intersection. The pattern continues and 
at the $(2 k+1)$-stage Lemma 4.8 gives the compatibility

$$
\delta i_{\alpha_{0} \cdots \hat{\alpha}_{i} \cdots \alpha_{2 k+1}}^{*} \operatorname{CS}_{\alpha_{0} \cdots \hat{\alpha}_{i} \cdots \alpha_{2 k+1}}(\eta)=h_{\alpha_{0} \cdots \alpha_{2 k+1}} \cdot \operatorname{CS}_{\alpha}^{\prime}(\eta) .
$$

From the universal property for $\infty$-limits, such a cone gives rise to a canonical map

$$
\mathcal{H}\left(\Omega^{*}\left[u, u^{-1}\right], d_{H}\right) \rightarrow \lim \left\{\left.\left.\ldots \xi \bigvee_{\alpha \beta} \mathcal{H}\left(\Omega^{*}\left[u, u^{-1}\right]\right)\right|_{U_{\alpha \beta}} \sum_{i_{\alpha}^{*}}^{i_{\beta}^{*}} \bigvee_{\alpha} \mathcal{H}\left(\Omega^{*}\left[u, u^{-1}\right]\right)\right|_{U_{\alpha}}\right\}
$$

Moreover, since the maps $\mathrm{CS}_{\alpha}(\eta)$ are local equivalences, it follows from descent that this map is an equivalence.

Now consider the canonical inclusion $\iota: \mathbb{R}\left[u, u^{-1}\right] \hookrightarrow \Omega^{*}\left[u, u^{-1}\right]$ induces a morphism of sheaves of spectra

$$
\iota: \mathcal{H}\left(\mathbb{R}\left[u, u^{-1}\right]\right) \longrightarrow \mathcal{H}\left(\Omega^{*}\left[u, u^{-1}\right]\right) .
$$

Consider the induced homotopy commutative diagram

$$
\begin{gathered}
\left.\left.\left.\ldots \sum \bigvee_{\alpha \beta \gamma} \mathcal{H}\left(\mathbb{R}\left[u, u^{-1}\right]\right)\right|_{U_{\alpha \beta \gamma}} \sum \bigvee_{\alpha \beta} \mathcal{H}\left(\mathbb{R}\left[u, u^{-1}\right]\right)\right|_{U_{\alpha \beta}} \sum_{i_{\alpha}^{*}}^{i_{\beta}^{*}} \bigvee_{\alpha} \mathcal{H}\left(\mathbb{R}\left[u, u^{-1}\right]\right)\right|_{U_{\alpha}} \\
\downarrow^{\iota} \\
\left.\left.\left.\cdots \sum \bigvee_{\alpha \beta \gamma} \mathcal{H}\left(\Omega^{*}\left[u, u^{-1}\right]\right)\right|_{U_{\alpha \beta \gamma}} \sum \bigvee_{\alpha \beta} \mathcal{H}\left(\Omega^{*}\left[u, u^{-1}\right]\right)\right|_{U_{\alpha \beta}} \sum_{i_{\alpha}^{*}}^{i_{\beta}^{*}} \bigvee_{\alpha} \mathcal{H}\left(\Omega^{*}\left[u, u^{-1}\right]\right)\right|_{U_{\alpha}}
\end{gathered}
$$

where again the cup product with cocycle $h_{\alpha_{0} \cdots \alpha_{2 k+1}}$ gives a nontrivial homotopy filling the top cosimplicial diagram via the corresponding map

$$
h_{\alpha_{0} \cdots \alpha_{2 k+1}}: \Delta^{2 k+1} \longrightarrow \operatorname{Map}\left(\left.\mathcal{H}\left(\mathbb{R}^{*}\left[u, u^{-1}\right]\right)\right|_{U_{\alpha}},\left.\mathcal{H}\left(\mathbb{R}^{*}\left[u, u^{-1}\right]\right)\right|_{U_{\alpha_{0} \cdots \alpha_{2 k+1}}}\right) .
$$

The diagram manifestly commutes up to homotopy (with degenerate homotopies filling the squares). This induces a map on corresponding $\infty$-limits, which is an equivalence since $\iota$ is an equivalence at each stage. By construction, we identify the top limit with the module spectrum $\mathcal{H}\left(\mathbb{R}\left[u, u^{-1}\right]\right)_{h}$. Combining this with the equivalence constructed above, we have a zig-zag of equivalences

$$
\mathcal{H}\left(\mathbb{R}\left[u, u^{-1}\right]\right) \stackrel{\sim}{\longrightarrow} \lim _{i \in \Delta^{o p}}\left(\mathcal{H}\left(\left.\Omega^{*}\left[u, u^{-1}\right]\right|_{\alpha_{0} \cdots \alpha_{i}}\right)\right) \stackrel{\sim}{\sim} \mathcal{H}\left(\Omega^{*}\left[u, u^{-1}\right], d_{H}\right),
$$

which gives rise to the desired equivalence.

Remark 4.10. The equivalence (4.4) depends on the choice of Čech-de Rham cochain trivializing the difference $H-h_{\alpha_{0} \cdots \alpha_{2 k+1}}$. Such a cochain is an element in degree 1 of the totalization $\operatorname{Tot}^{\bullet}\left(\left\{U_{\alpha}\right\} ; \Omega_{\mathrm{cl}}^{\leqslant 2 k+1}\right)$. Given the previous construction, it is tedious but straight forward to check further that elements of degree 2 gives rise to zig-zags between zig-zags (a 2-simplex in $\operatorname{Pic}_{\mathrm{dR}}(M)$ ), so on and so forth.

Theorem 4.11 (Twisting periodic Deligne cohomology by odd degree gerbes with connection). Let $\widehat{\mathrm{Tw}}(M)$ denote the stack of twists, evaluated on a smooth manifold $M$. Then every Čech-Deligne cocycle of degree $2 k+1$ defines a twist of periodic Deligne cohomology. In fact, there is a morphism of smooth stacks

$$
\mathbf{B}^{2 k} U(1)_{\nabla} \longrightarrow \widehat{\mathrm{Tw}}
$$

refining the map $K(\mathbb{Z}, 2 k+1) \rightarrow B \mathrm{GL}_{1}\left(\mathcal{H} \mathbb{Z}\left[u, u^{-1}\right]\right) \hookrightarrow \operatorname{Pic}_{\mathcal{H} \mathbb{Z}\left[u, u^{-1}\right]}^{\text {top }}$. 
Proof. The stack $\mathbf{B}^{2 k} U(1)_{\nabla}$ fits into the Cartesian square (4.1). This pullback in smooth stacks can be computed by the stackification of the corresponding pullback in prestacks, which is computed objectwise. Moreover, a morphism of prestacks into a stack is, equivalently, a morphism of stacks out of the stackification. ${ }^{12}$ It therefore suffices to construct the map objectwise out of the three stacks $\Omega_{\mathrm{cl}}^{2 k+1}, \mathbf{B}^{2 k+1} \mathbb{Z}$ and $\Omega_{\mathrm{cl}}^{\leqslant 2 k+1}$ and for every (1-)homotopy filling the diagram, a corresponding homotopy filling diagram (1.2). To that end, fix an arbitrary manifold $M$ and define the homotopy commutative diagram

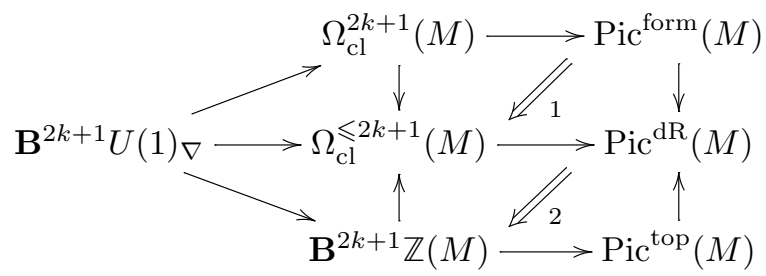

as follows. The top horizontal map in the diagram sends a closed odd-degree form to the invertible module over the periodic de Rham complex, $\left(\Omega^{*}\left[u, u^{-1}\right](M), d_{H}\right)$, where the differential $d_{H}=d+H \wedge$ acts on a differential form as

$$
d_{H}(\omega)=d_{H}\left(\omega_{0}+\omega_{2}+\cdots\right)=d \omega_{0}+d \omega_{2}+\cdots+\left(H \wedge \omega_{0}+d \omega_{2 k}\right)+\cdots .
$$

The middle horizontal takes a Cech de Rham cochain and maps it to the $\infty$-limit over the corresponding cosimplicial diagram

$$
\left.\left.\left.\cdots \sum \mathcal{H}\left(\Omega^{*}\left[u, u^{-1}\right]\right)\right|_{U_{\alpha \beta \gamma}} \sum \mathcal{H}\left(\Omega^{*}\left[u, u^{-1}\right]\right)\right|_{U_{\alpha \beta}} \sum_{i_{\beta}^{*}}^{i_{\alpha}^{*}} \mathcal{H}\left(\Omega^{*}\left[u, u^{-1}\right]\right)\right|_{U_{\alpha}}
$$

considered in the discussion preceding the theorem. The bottom horizontal map assigns an integral Čech cocycle in degree $(2 k+1)$ to the sheaf of module spectra $\mathcal{H}\left(\mathbb{Z}\left[u, u^{-1}\right]\right)_{h}$, where the simplicial maps are completely determined by

$$
h_{\alpha_{0} \cdots \alpha_{2 k+1}}: \Delta^{2 k+1} \longrightarrow \operatorname{Map}\left(\mathcal{H}\left(\left.\mathbb{Z}\left[u, u^{-1}\right]\right|_{U_{\alpha_{0} \cdots \alpha_{2 k+1}}},\left.\mathbb{Z}\left[u, u^{-1}\right]\right|_{U_{\alpha_{0} \cdots \alpha_{2 k+1}}}\right)\right) .
$$

Then the homotopies 1 and 2 are given by the two equivalences in the zig-zag (4.4), the homotopy labeled by 1 (respectively, 2) given by the equivalence on the right (resp., left). By the universal property, we have an induced map $\mathbf{B}^{2 k+1} U(1)_{\nabla} \rightarrow \widehat{\mathrm{Tw}}$.

Remark 4.12 (Bundle of spectra approach). Twisting periodic Deligne cohomology via gerbes can also be described using the framework of bundles of spectra. We now sketch this construction (which can be regarded as dual to the approach we have taken). Consider the sheaf of complexes $\left(\Omega^{*}\left[u, u^{-1}\right], d_{H}\right)$ on a smooth manifold $M$, which is degreewise identical to the periodic complex of forms, but which is equipped with the differential $d_{H}:=d+H \wedge$, acting by (4.5). Here, $H$ a closed form of degree $2 k+1$. Applying the Eilenberg-MacLane functor $\mathcal{H}$ to $\left(\Omega^{*}\left[u, u^{-1}\right], d_{H}\right)$ gives a sheaf of spectra on $M$. Now $\left(\Omega^{*}\left[u, u^{-1}\right], d_{H}\right)$ is an invertible module over $\left(\Omega^{*}\left[u, u^{-1}\right], d\right)$ which is locally equivalent (by the Poincaré Lemma) to the constant sheaf $\mathbb{R}\left[u, u^{-1}\right]$.

\footnotetext{
${ }^{12}$ This follows from the adjunction $i \vdash L$, with $L$ the stackification functor and $i$ the inclusion functor.
} 
Thus $\mathcal{H}\left(\Omega^{*}\left[u, u^{-1}\right], d_{H}\right)$ gives a sheaf of spectra which is a module over $\mathcal{H} \mathbb{R}\left[u, u^{-1}\right]$. Pulling back the universal bundle of spectra

$$
\lambda \longrightarrow \operatorname{Pic}_{\mathcal{H} \mathbb{R}\left[u, u^{-1}\right]}^{\mathrm{dR}}
$$

(see [43] for this construction) by the map $\tau: M \rightarrow \operatorname{Pic}_{\mathcal{H} \mathbb{R}\left[u, u^{-1}\right]}^{\mathrm{dR}}$, which picks out the twisted sheaf of spectra $\mathcal{H}\left(\Omega^{*}\left[u, u^{-1}\right], d_{H}\right)$, gives a smooth bundle of spectra $E \rightarrow M$. The sheaf of local sections of the latter evaluated on $U$ is, by definition, $\mathcal{H}\left(\Omega^{*}\left[u, u^{-1}\right], d_{H}\right)(U)$. Choose local potentials $B_{\alpha}$ for $H$ on each element of a good open cover $\left\{U_{\alpha}\right\}$ of $M$ (i.e. $d B_{\alpha}=H$ ). Then, on each patch $U_{\alpha}$, we have quasiisomorphisms of sheaves of complexes

$$
e^{B_{\alpha}} \wedge:\left.\left.\left(\Omega^{*}\left[u, u^{-1}\right], d_{H}\right)\right|_{U_{\alpha}} \stackrel{\simeq}{\longrightarrow} \Omega^{*}\left[u, u^{-1}\right]\right|_{U_{\alpha}},
$$

which send a local section $\omega$ to the wedge product with the formal exponential

$$
e^{B_{\alpha}}=1+B_{\alpha}+\frac{1}{2 !} B_{\alpha}^{2}+\cdots
$$

These quasi-isomorphisms correspond to local trivializations

$$
e^{B_{\alpha}} \wedge:\left.\mathcal{H}\left(\Omega^{*}\left[u, u^{-1}\right]\right)\right|_{U_{\alpha}} \times\left. U_{\alpha} \longrightarrow E\right|_{U_{\alpha}} .
$$

In fact, dual to the discussed above, a choice of representative of $H$ in the Čech-de Rham double complex gives rise to an $\infty$-cocone

$$
\left.\cdots \gtreqless \coprod_{\alpha \beta} \mathcal{H}\left(\Omega^{*}\left[u, u^{-1}\right]\right)\right|_{U_{\alpha \beta}} \times\left. U_{\alpha \beta} \rightrightarrows \coprod_{\alpha} \mathcal{H}\left(\Omega^{*}\left[u, u^{-1}\right]\right)\right|_{U_{\alpha}} \times U_{\alpha} \cdots>E,
$$

where the homotopies at each stage are determined by the Čech-de Rham data for $H$. By descent, one concludes that $E$ is, in fact, an $\infty$-colimit over this diagram. Notice also that, since the global sections of $E$ are $\mathcal{H}\left(\Omega^{*}\left[u, u^{-1}\right](M), d_{H}\right)$, we immediately have that the twisted cohomology represented by the bundle $E$ is the $H$-twisted de Rham cohomology of $M$. If $H$ has integral periods, then working dually as in the proof of Theorem 4.11, we see that the pullback of the universal bundle $\lambda \rightarrow \widehat{\mathrm{Tw}}$ by a gerbe $\hat{h}: M \rightarrow \mathbf{B}^{2 k+1} U(1)_{\nabla} \rightarrow \widehat{\mathrm{Tw}}$ gives a bundle of spectra whose sections are the module spectrum represented by the twist.

Definition 4.13 (Twisted periodic Deligne cohomology). Let $H$ be a closed differential form of odd degree which has integral periods. Then $H$ can be lifted to

$$
\hat{h}: M \longrightarrow \mathbf{B}^{2 k} U(1)_{\nabla} \text {. }
$$

According to Proposition 4.5, we can regard this as either twisting $\mathcal{H}(\mathcal{D}(\mathrm{ev}))$ or $\mathcal{H}(\mathcal{D}($ odd $))$. Let $\mathcal{Z}_{\hat{h}}^{\text {ev }} \rightarrow M$ and $\mathcal{Z}_{\hat{h}}^{\text {odd }} \rightarrow M$ be the corresponding smooth bundles of spectra. We define the twisted periodic Deligne cohomology to be the homotopy classes of sections of the corresponding bundle $\mathcal{Z}_{\hat{h}}^{\text {ev / odd }} \rightarrow M$, i.e.,

$$
\widehat{H}^{\mathrm{ev}}(M ; \hat{h}):=\pi_{0} \Gamma\left(M ; \mathcal{Z}_{\hat{h}}^{\mathrm{ev}}\right) \quad \text { and } \quad \widehat{H}^{\text {odd }}(M ; \hat{h}):=\pi_{0} \Gamma\left(M ; \mathcal{Z}_{\hat{h}}^{\text {odd }}\right) .
$$

Just as one can define tensor product and direct sum of vector bundles, one can similarly define the wedge product and smash product of bundles of spectra (see [43] for the definition of the smash product; the wedge product is defined similarly). In the present case, we have a $\mathbb{Z} / 2$-graded bundle of spectra $E^{\mathrm{ev}} \vee E^{\text {odd }} \rightarrow M$. The local sections of this bundle are given by evaluating the wedge product of spectra 
$\mathcal{H}(\mathcal{D}($ ev $)) \vee \mathcal{H}(\mathcal{D}($ odd $)) \simeq \mathcal{H}(\mathcal{D}($ ev $) \oplus \mathcal{D}($ odd $))$ on open subsets $U \subset M$. Given the multiplicative structure of periodic Deligne cohomology from Section 3.2, we have:

Proposition 4.14 (Module structure of twisted periodic Deligne cohomology). The sheaf of sections of the bundle $E^{\mathrm{ev}} \vee E^{\mathrm{odd}} \rightarrow M$ is a module spectrum over the sheaf of ring spectra given by $\mathcal{H}(\mathcal{D}(\mathrm{ev})) \vee \mathcal{H}(\mathcal{D}(\mathrm{odd}))$. The module action descends to

$$
\mu: \widehat{H}^{\mathrm{ev} / \text { odd }}\left(M ; \mathbb{Z}\left[u, u^{-1}\right]\right) \otimes \widehat{H}^{\mathrm{ev} / \text { odd }}(M ; \hat{h}) \longrightarrow \widehat{H}^{\mathrm{ev} / \text { odd }}(M ; \hat{h}),
$$

turning $\widehat{H}^{\mathrm{ev} / \text { odd }}(M ; \hat{h})$ into a module over the superalgebra $\widehat{H}^{\mathrm{ev} / \text { odd }}\left(M ; \mathbb{Z}\left[u, u^{-1}\right]\right)$.

\subsection{Properties of twisted periodic smooth Deligne cohomology}

In this section, we list several properties of twisted periodic Deligne cohomology.

Proposition 4.15 (Properties of twisted periodic Deligne cohomology). Let $M$ be a smooth manifold and fix a twist $\hat{h}: M \rightarrow \mathbf{B}^{2 k} U(1)_{\nabla}$. Consider the category of such pairs $(M, \hat{h})$, with morphisms $(f, \phi):(M, \hat{h}) \rightarrow(M, \hat{\ell})$ given by smooth maps $f: M \rightarrow$ $N$ and equivalences $\phi: f^{*} \hat{\ell} \Rightarrow \hat{h}$. The assignment $(M, \hat{h}) \mapsto \widehat{H}^{*}(M ; \hat{h})$ satisfies:

1. $\widehat{H}^{*}(M ; \hat{h})$ is functorial with respect to the maps $f:(M, \hat{h}) \rightarrow(N, \hat{\ell})$.

2. The functor $\widehat{H}^{*}(-; \hat{h})$ satisfies the Eilenberg-Steenrod axioms (modulo the dimension axiom and homotopy invariance!) for a reduced cohomology theory. In particular, we have a Mayer-Vietoris sequence which takes the form

$$
\begin{gathered}
\cdots \longrightarrow H_{\mathbb{R} / \mathbb{Z}}^{*-2}(U ; h) \oplus H_{\mathbb{R} / \mathbb{Z}}^{*-2}(V ; h) \longrightarrow H_{\mathbb{R} / \mathbb{Z}}^{*-2}(U \cap V ; h) \\
\longrightarrow \widehat{H}^{*}(M ; \hat{h}) \longrightarrow \widehat{H}^{*}(U ; \hat{h}) \oplus \widehat{H}^{*+1}(V ; \hat{h}) \longrightarrow \widehat{H}^{*}(U \cap V ; \hat{h}) \\
\longrightarrow H^{*+1}(M ; h) \longrightarrow H^{*+1}(U ; h) \oplus H^{*+1}(V ; h) \longrightarrow \cdots
\end{gathered}
$$

3. For $\hat{h}: M \rightarrow \mathbf{B}^{2 k} U(1)_{\nabla}$ a trivial twist (i.e. $\hat{h} \simeq *$ in smooth stacks) we have

$$
\widehat{H}^{*}(M ; \hat{h}) \cong \widehat{H}^{*}\left(M ; \mathbb{Z}\left[u, u^{-1}\right]\right) .
$$

Even more strongly, we still have an isomorphism (4.6) if just the underlying topological twist $h: M \rightarrow K(\mathbb{Z}, 2 k+1)$ is trivial.

Proof. (i) Given a map $f: M \rightarrow N$ satisfying the desired compatibility, we have an induced double pullback diagram

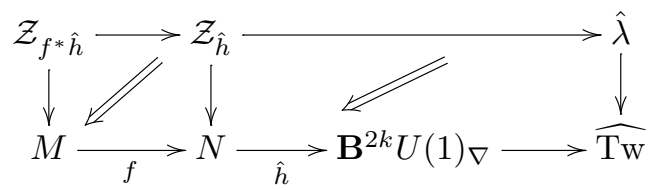

and $\phi$ induces the identification $\mathcal{Z}_{f^{*} \hat{h}} \simeq \mathcal{Z}_{\hat{\ell}}$. As a consequence, we have an induced morphism of sections $f^{*}: \Gamma\left(N ; \mathcal{Z}_{\hat{h}}\right) \rightarrow \Gamma\left(M ; \mathcal{Z}_{\hat{\ell}}\right)$. Passing to homotopy groups yields a map $f^{*}: \widehat{H}^{*}(N ; \hat{\ell}) \rightarrow \widehat{H}^{*}(M ; \hat{h})$. 
(ii) Additivity. Let $M=\coprod_{\alpha} M_{\alpha}$ with each $M_{\alpha}$ a smooth manifold. A map $\hat{h}: M \rightarrow$ $\mathbf{B}^{2 k} U(1)_{\nabla}$ is equivalently a collection of maps $h_{\alpha}: M_{\alpha} \rightarrow \mathbf{B}^{2 k} U(1)_{\nabla}$. Then the spectrum of sections $\Gamma\left(M, \mathcal{Z}_{\hat{h}}\right)$ splits as a product $\prod_{\alpha} \Gamma\left(M_{\alpha}, \mathcal{Z}_{\hat{h}_{\alpha}}\right)$. Since taking homotopy groups commutes with products, we have an isomorphism $\widehat{H}^{*}(M, \hat{h}) \cong$ $\prod_{\alpha} \widehat{H}^{*}\left(M_{\alpha}, \hat{h}_{\alpha}\right)$.

Exactness. This follows verbatim as in proof of Proposition 2.8, with the space $X$ replaced by a smooth manifold $M, A \subset M$ a submanifold, and the map $h: X \rightarrow$ $K(\mathbb{Z}, 2 k+1)$ replaced by the refinement $\hat{h}: M \rightarrow \mathbf{B}^{2 k} U(1)_{\nabla}$.

(iii) Finally, if the twist $\hat{h}: M \rightarrow \mathbf{B}^{2 k} U(1)_{\nabla}$ is topologically trivial, i.e., its geometric realization $h:|M| \simeq M \rightarrow\left|\mathbf{B}^{2 k} U(1)_{\nabla}\right| \simeq K(\mathbb{Z}, 2 k+1)$ is homotopic to the constant map induced by $0 \rightarrow \mathbb{Z}$. In this case, the underlying twisted spectrum $\mathcal{H} \mathbb{Z}[u$, $\left.u^{-1}\right]_{h}$ is equivalent to $\mathcal{H} \mathbb{Z}\left[u, u^{-1}\right]$ and we have the diagram

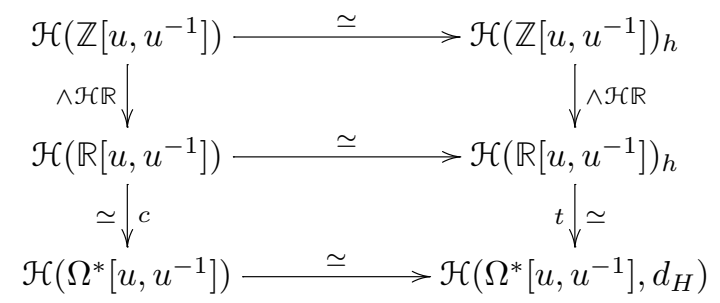

where the bottom equivalence depends on a choice of homotopy inverse for $c$ and is defined as the obvious composition in the diagram. By the basic properties of the functor $\mathcal{H}$ (see [15, pp. 17-18] for discussion), the existence of the bottom equivalence implies that $\Omega^{*}\left[u, u^{-1}\right]$ and $\left(\Omega^{*}\left[u, u^{-1}\right], d_{H}\right)$ are connected by a zig-zag of quasiisomorphisms. This is manifestly the data needed to define an equivalence in the $\infty$-groupoid $\widehat{\mathrm{Tw}}(M)$.

\section{The spectral sequence $\widehat{\mathrm{AHSS}}_{h}$ and examples}

In this section, we apply the twisted Atiyah-Hirzebruch spectral sequence (both the classical $[66,67,4]$ and the differential refinement $[43])$ to calculate the twisted periodic integral and Deligne cohomology of spheres.

\subsection{The sequence in twisted periodic smooth Deligne cohomology}

In [4], the first nonvanishing differential for the twisted AHSS (applied to $K$ theory) on a space $X$ was identified by observing that the only degree three increasing operations for spaces equipped with maps $X \rightarrow K(\mathbb{Z}, 3)$ are given by

$$
H^{n+3}(K(\mathbb{Z}, n) \times K(\mathbb{Z}, 3)) \cong H^{n+3}(K(\mathbb{Z}, n)) \oplus H^{n+3}(K(\mathbb{Z}, 3)) \oplus \mathbb{Z} .
$$

The third factor on the right hand side is generated by the product of the generators for $H^{n}(K(\mathbb{Z}, n))$ and $H^{3}(K(\mathbb{Z}, 3))$. From this, one deduces that

$$
d_{3}(x)=S q_{\mathbb{Z}}^{3}(x)-[h] \cup x,
$$

with $[h]$ the twisting integral class and $S q_{\mathbb{Z}}^{3}$ the third integral Steenrod square, which comes from the untwisted AHSS for $K$-theory [3]. 
For integral cohomology, considering again a degree three twist $h$, we then find that $^{13} d_{3}(x)=-[h] \cup x$. The same argument applies not only in the degree three case, but also in higher odd degrees. This is due to the fact that for spaces equipped with maps $X \rightarrow K(2 k+1 ; \mathbb{Z})$, we again have the identification

$H^{n+2 k+1}(K(\mathbb{Z}, n) \times K(\mathbb{Z}, 2 k+1)) \cong H^{n+2 k+1}(K(\mathbb{Z}, n)) \oplus H^{n+2 k+1}(K(\mathbb{Z}, 2 k+1)) \oplus \mathbb{Z}$, with the last factor being generated by the product of the generator of $H^{n}(K(\mathbb{Z}, n))$ and the generator of $H^{2 k+1}(K(\mathbb{Z}, 2 k+1))$. We, therefore, have the following.

Proposition 5.1 (First differential for $\mathrm{AHSS}_{h}$ for twisted periodic integral cohomology). Let $h: X \rightarrow K(\mathbb{Z}, 2 k+1)$ be a twist of periodic integral cohomology. Then the first nonvanishing differential in the associated AHSS occurs on the $E_{2 k+1}$-page and is given by $d_{2 k+1}(x)=-[h] \cup x$.

We will illustrate this in Examples 5.5 and 5.6 below. In [43], we developed an AHSS for twisted differential cohomology theories, in turn generalizing that of a differential theory [42]. In the case of periodic Deligne cohomology, there are two spectral sequences corresponding to the even and odd degrees (separately).

Lemma 5.2 (The $E_{2}$-page for even degrees in $\widehat{\operatorname{AHSS}}_{\hat{h}}$ for twisted periodic Deligne cohomology). The $E_{2}$-page for the even case looks as follows:

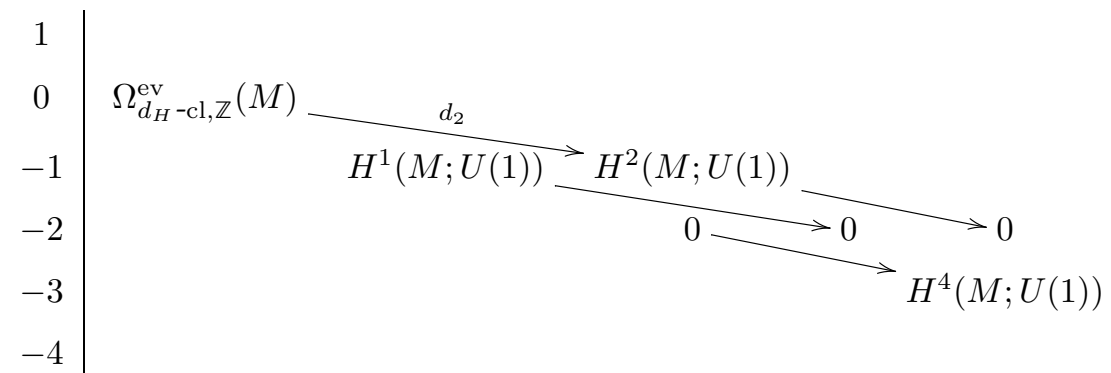

where $\Omega_{d_{H}-\mathrm{cl}, \mathbb{Z}}^{\mathrm{ev}}(M)$ is the subgroup of those even forms on $M$ which are twisted-closed and whose degree zero component is given by an integer, i.e. $\omega=n_{0}+\omega_{2}+\omega_{4}+\cdots$.

Lemma 5.3 (The $E_{2}$-page for odd degrees in $\widehat{\operatorname{AHSS}}_{\hat{h}}$ for twisted periodic Deligne cohomology). The spectral sequence for the odd degrees looks as follows:

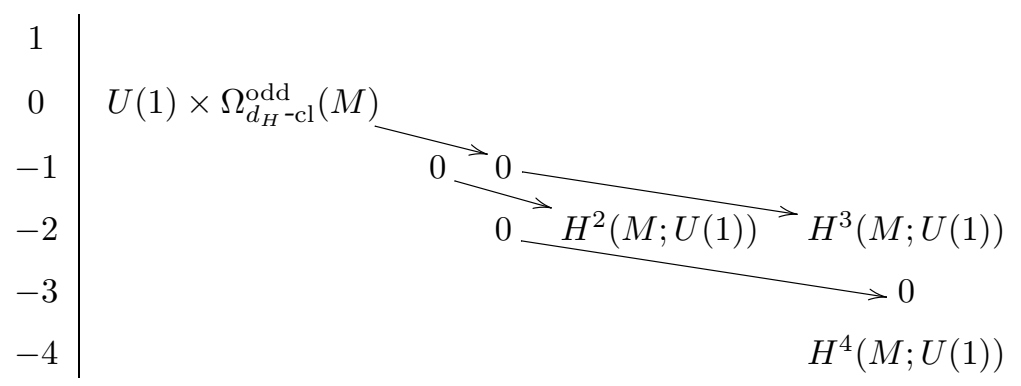

where $\Omega_{d_{H} \text {-cl }}^{\text {odd }}(M)$ is the group of twisted-closed odd forms on $M$.

\footnotetext{
${ }^{13}$ This can be deduced, for example, from the $K$-theory differential and the fact that (on spheres) the Chern character lands in integral cohomology.
} 
For twisted differential $K$-theory, in [43] we identified the first nonzero differential in the spectral sequence as

$$
d_{3}(x)=\widehat{S q}_{\mathbb{Z}}^{3}(x)+[\hat{h}] \cup_{\mathrm{DB}} x,
$$

where $\widehat{S q}_{\mathbb{Z}}^{3}$ is a torsion operation in differential cohomology inherited from $S q^{3}$ (see [41]), and $[\hat{h}] \cup_{\mathrm{DB}}(-)$ is the Deligne-Beilinson cup product operation. The same argument used in [43, Proposition 25] applies to the case of differential refinements of the higher degree twists for periodic integral cohomology. As a result we have:

Proposition 5.4 (First differential for $\widehat{\mathrm{AHSS}}_{\hat{h}}$ for twisted Deligne cohomology). Let $h: M \rightarrow \mathbf{B}^{2 k} U(1)_{\nabla}$ be a twist of periodic Deligne cohomology. Then the differential in the associated AHSS on the $E_{2 k+1}-$ page $e^{14}$ is given by $d_{2 k+1}(x)=-[\hat{h}] \cup_{\mathrm{DB}} x$.

We will illustrate this in Examples 5.7 and 5.8 below.

\subsection{Examples via the spectral sequence}

We now proceed with our examples illustrating the AHSS that we developed in Section 5.1 to both twisted periodic integral cohomology (Section 2) and twisted periodic Deligne cohomology (Section 4).

Example 5.5 (Twisted periodic integral cohomology of even spheres). For even spheres, the class of the twist vanishes for parity reasons. Therefore, the AHSS degenerates at the $E_{2}$-page and we immediately identify

$$
H^{\mathrm{ev}}\left(S^{2 k} ; \mathbb{Z}\left[u, u^{-1}\right]\right)=\mathbb{Z} \oplus \mathbb{Z} \quad \text { and } \quad H^{\text {odd }}\left(S^{2 k}, \mathbb{Z}\left[u, u^{-1}\right]\right)=0 .
$$

Example 5.6 (Twisted periodic integral cohomology of odd spheres). For an odddimensional sphere $S^{2 k+1}$, the only interesting twist occurs in degree $2 k+1$. Consequently, the only nonzero differential in the AHSS occurs on the $E_{2 k+1}$-page, giving the sequence $\mathbb{Z} \stackrel{-[h] \cup}{\longrightarrow} H^{2 k+1}\left(S^{2 k+1} ; \mathbb{Z}\right) \cong \mathbb{Z} \rightarrow 0$. Thus, with $h$ also denoting the integer corresponding to the topological twist $h$, we get

$$
H^{\text {odd }}\left(S^{2 k+1} ; \mathbb{Z}\left[u, u^{-1}\right]\right) \cong \mathbb{Z} / h \quad \text { and } \quad H^{\mathrm{ev}}\left(S^{2 k+1} ; \mathbb{Z}\left[u, u^{-1}\right]\right) \cong 0 .
$$

Example 5.7 (Twisted periodic Deligne cohomology of even spheres). Let $\hat{h}: M \rightarrow$ $\mathbf{B}^{2 k} U(1)_{\nabla}$ be a twist for periodic Deligne cohomology. For parity reasons, the class of the underlying topological twist $h \in H^{2 k+1}\left(S^{2 k} ; \mathbb{Z}\right)$ vanishes. By property (iii) of Proposition 4.15, it follows that we have an isomorphism

$$
\widehat{H}^{\text {ev } / \text { odd }}\left(S^{2 k} ; \hat{h}\right) \cong \widehat{H}^{\text {ev/odd }}\left(S^{2 k} ; \mathbb{Z}\left[u, u^{-1}\right]\right),
$$

with the underlying untwisted theory. We computed the corresponding groups earlier in Example 3.7 (Section 3.2), which immediately yields

$$
\widehat{H}^{\mathrm{ev}}\left(S^{2 k} ; \hat{h}\right)=\Omega^{\text {odd }}\left(S^{2 k}\right) / \operatorname{im}(d) \oplus \mathbb{Z} \oplus \mathbb{Z} \text { and } \widehat{H}^{\text {odd }}\left(S^{2 k}, \hat{h}\right)=\Omega^{\mathrm{ev}}\left(S^{2 k}\right) / \Omega_{\mathrm{cl}, \mathbb{Z}}^{\mathrm{ev}}\left(S^{2 k}\right) \text {. }
$$

The case of odd spheres is more involved.

${ }^{14}$ Note that there is also a differential on the $E_{2 k}$-page; but we do not use this. 
Example 5.8 (Twisted periodic Deligne cohomology of odd spheres). For the odd spheres, the only interesting twist are the differential refinements of the topological twists $[h] \in H^{2 k+1}\left(S^{2 k+1} ; \mathbb{Z}\right)$. Choose such a differential refinement $\hat{h}: M \rightarrow \mathbf{B}^{2 k} U(1)_{\nabla}$. Then the spectral sequence has one nontrivial differential

$$
d_{2 k+1}: U(1) \times \Omega_{d_{H^{-c l}}}^{\text {odd }}\left(S^{2 k+1}\right) \longrightarrow U(1) \cong H^{2 k+1}\left(S^{2 k+1} ; U(1)\right),
$$

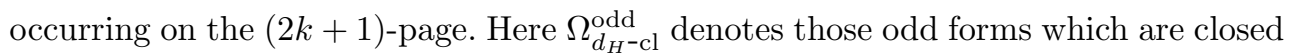
under the twisted differential $d_{H}$. The restriction to the factor $U(1)$ is given by the Deligne-Beilinson cup product with $[\hat{h}]$. This can be computed as follows. As above, let $h$ be the integer representing the topological class $h \in \mathbb{Z}$. For $\theta \in U(1)$, we have $[\hat{h}] \cup_{\mathrm{DB}} \theta=h \theta$. The kernel of $d_{2 k+1}$ restricted to this factor is the subgroup of $h$ roots of unity which is isomorphic to $\mathbb{Z} / h$. Since the map $\theta \mapsto h \theta$ is surjective the First Isomorphism Theorem implies that the factor $\Omega_{d_{H-c l}}^{\text {odd }}\left(S^{2 k+1}\right)$ is killed by $d_{2 k+1}$. It remains to solve the extension problem

$$
0 \longrightarrow \mathbb{Z} / h \longrightarrow \widehat{H}^{\text {odd }}\left(S^{2 k+1} ; \hat{h}\right) \longrightarrow \Omega_{d_{H^{-c l}}}^{\text {odd }}\left(S^{2 k+1}\right) \longrightarrow 0 \text {. }
$$

Now for any abelian group $A$ and any divisible group $B$ the $\operatorname{Ext} \operatorname{group} \operatorname{Ext}(A, B)$ vanishes. Since the group $\Omega_{d_{H} \text {-cl }}^{\text {odd }}\left(S^{2 k+1}\right)$ is divisible, we then have

$$
\operatorname{Ext}^{1}\left(\mathbb{Z} / h, \Omega_{d_{H^{-c l}}}^{\text {odd }}\left(S^{2 k+1}\right)\right) \cong \Omega_{d_{H}-\mathrm{cl}}^{\text {odd }}\left(S^{2 k+1}\right) / h \Omega_{d_{H^{-c l}}}^{\text {odd }}\left(S^{2 k+1}\right) \cong 0 .
$$

Thus, the extension must be the trivial one and we conclude that

$$
\widehat{H}^{\text {odd }}\left(S^{2 k+1} ; \hat{h}\right) \cong \mathbb{Z} / h \oplus \Omega_{d_{H} \text {-cl }}^{\text {odd }}\left(S^{2 k+1}\right) .
$$

\section{Acknowledgments}

The authors thank the organizers and participants of the Geometric Analysis and Topology Seminar at the Courant Institute at NYU for asking about twisting Deligne cohomology, during a talk by H.S., which encouraged the authors to finish this twostage project, starting with [43]. We thank the referee for useful suggestions that improved the paper.

\section{References}

[1] M. Ando, A.J. Blumberg, D. Gepner, Twists of K-theory and TMF, Superstrings, Geometry, Topology, and $C^{*}$-Algebras, 27-63, Proc. Sympos. Pure Math., 81, Amer. Math. Soc., Providence, RI, 2010, [arXiv:1002.3004].

[2] M. Ando, A.J. Blumberg, D. Gepner, M.J. Hopkins, C. Rezk, Units of ring spectra, orientations and Thom spectra via rigid infinite loop space theory, J. Topol. 7 (2014), no. 4, 1077-1117.

[3] M.F. Atiyah, F. Hirzebruch, Vector bundles and homogeneous spaces, Proc. Sympos. Pure Math., vol. III, 7-38, Amer. Math. Soc., Providence, R.I., 1962.

[4] M. Atiyah, G. Segal, Twisted K-theory and cohomology, Inspired by S.S. Chern, 5-43, World Sci. Publ., Hackensack, NJ, 2006. 
[5] C. Bär, C. Becker, Differential Characters, Lecture Notes in Math., 2112, Springer, Cham, Switzerland, 2014.

[6] A. Beilinson, Higher regulators and values of L-functions, J. Soviet Math. 30 (1985), 2036-2070.

[7] P. Bouwknegt, A. Carey, V. Mathai, M. Murray, D. Stevenson, Twisted Ktheory and K-theory of bundle gerbes, Comm. Math. Phys. 228 (2002), 17-45.

[8] R. Bott, L.W. Tu, Differential Forms in Algebraic Topology, Springer-Verlag, New York, Berlin, 1982.

[9] J.-L. Brylinski, Loop Spaces, Characteristic Classes and Geometric Quantization, Progr. Math., 107, Birkhäuser, Boston, 1993.

[10] J.-L. Brylinski, D. McLaughlin, The geometry of degree-four characteristic classes and of line bundles on loop spaces I, Duke Math. J. 75 (1994), no. 3, 603-638.

[11] J.-L. Brylinski, D. McLaughlin, Čech cocycles for characteristic classes, Comm. Math. Phys. 178 (1996), 225-236.

[12] M. Bullejos, E. Faro, M.A. Garcia-Muñoz, Homotopy colimits and cohomology with local coefficients, Cah. Topol. Géom. Différ. Catég. 44 (2003), 63-80.

[13] U. Bunke, Differential Cohomology, [arXiv:math.AT/1208.3961].

[14] U. Bunke, M. Kreck, T. Schick, A geometric description of differential cohomology, Ann. Math. Blaise Pascal 17 (2010), no. 1, 1-16.

[15] U. Bunke, T. Nikolaus, Twisted Differential Cohomology, [arXiv:1406.3231].

[16] U. Bunke, T. Nikolaus, M. Völkl, Differential cohomology theories as sheaves of spectra, J. Homotopy Relat. Struct. 11 (2016), no. 1, 1-66.

[17] U. Bunke and T. Schick, Uniqueness of smooth extensions of generalized cohomology theories, J. Topol. 3 (2010), 110-156.

[18] U. Bunke, T. Schick, M. Spitzweck, Sheaf theory for stacks in manifolds and twisted cohomology for $S^{1}$-gerbes, Algebr. Geom. Topol. 7 (2007), 1007-1062.

[19] J. Carlson, R. Hain, Extensions of variations of mixed Hodge structure, Astérisque 179-180 (1989), no. 9, 39-65.

[20] A.L. Carey, S. Johnson, M.K. Murray, D. Stevenson, B.-L. Wang, Bundle gerbes for Chern-Simons and Wess-Zumino-Witten theories, Comm. Math. Phys. 259 (2005), 577-613.

[21] J. Cheeger, J. Simons, Differential Characters and Geometric Invariants, 5080, Lecture Notes in Math., 1167, Springer, Berlin, 1985.

[22] P. Deligne, Théorie de Hodge: II, Publ. Math. Inst. Hautes Études Sci. 40 (1971), 5-57.

[23] J.L. Dupont, R. Ljungmann, Integration of simplicial forms and Deligne cohomology, Math. Scand. 97 (2005), 11-39.

[24] H. Esnault, E. Viehweg, Deligne-Beilinson cohomology, Beilinson's Conjectures on Special Values of L-Functions, 43-91, Academic Press, Boston, MA, 1988.

[25] D. Fiorenza, H. Sati, U. Schreiber, Extended higher cup-product Chern-Simons theory, J. Geom. Phys. 74 (2013), 130-163, [arXiv:1207.5449]. 
[26] D. Fiorenza, H. Sati, U. Schreiber, Multiple M5-branes, string 2-connections, and 7d nonabelian Chern-Simons theory, Adv. Theor. Math. Phys. 18 (2014), no. 2, 229-321, [arXiv:1201.5277].

[27] D. Fiorenza, H. Sati, U. Schreiber, A Higher stacky perspective on ChernSimons theory, Mathematical Aspects of Quantum Field Theories (Damien Calaque and Thomas Strobl eds.), Springer, Berlin, 2015, [arXiv:1301.2580].

[28] D. Fiorenza, H. Sati, U. Schreiber, The $E_{8}$ moduli 3-stack of the $C$-field in Mtheory, Comm. Math. Phys. 333 (2015), no. 1, 117-151, [arXiv:1202.2455].

[29] D. Fiorenza, U. Schreiber, J. Stasheff, Čech cocycles for differential characteristic classes - An infinity-Lie theoretic construction, Adv. Theor. Math. Phys. 16 (2012), 149-250, [arXiv:1011.4735] [math.AT].

[30] D.S. Freed, Dirac Charge Quantization and Generalized Differential Cohomology, 129-194, Surv. Differ. Geom., 7, Int. Press, Somerville, MA, 2000.

[31] D.S. Freed, The Verlinde algebra is twisted equivariant K-theory, Turkish J. Math. 25 (2001), 159-167.

[32] D.S. Freed, Classical Chern-Simons theory II, Special issue for S.S. Chern, Houston J. Math. 28 (2002), no. 2, 293-310.

[33] D.S. Freed, M. Hopkins, On Ramond-Ramond fields and K-theory, J. High Energy Phys. 5 (2000), 44, 14 pages.

[34] D.S. Freed, J. Lott, An index theorem in differential K-theory, Geom. Topol. 14 (2010), no. 2, 903-966.

[35] P. Gajer, Geometry of Deligne cohomology, Invent. Math. 127 (1997), 155-207.

[36] L. Gallot, E. Pilon, F. Thuillier, Higher dimensional abelian Chern-Simons theories and their link invariants, J. Math. Phys. 54 (2013), 022305.

[37] H. Gillet, Deligne homology and Abel-Jacobi maps, Bull. Amer. Math. Soc. 10 (1984), 285-288.

[38] K. Gomi, The formulation of the Chern-Simons action for general compact Lie groups using Deligne cohomology, J. Math. Sci. Univ. Tokyo 8 (2001), 223-242.

[39] K. Gomi, Central extensions of gauge transformation groups of higher abelian gerbes, J. Geom. Phys. 56 (2006), no. 9, 1767-1781.

[40] K. Gomi, U. Terashima, Chern-Weil construction for twisted K-theory, Comm. Math. Phys. 299 (2010), no. 1, 225-254.

[41] D. Grady, H. Sati, Primary operations in differential cohomology, Adv. Math. 335 (2018), no. 7, 519-562.

[42] D. Grady, H. Sati, Spectral sequences in smooth generalized cohomology, Algebr. Geom. Topol. 17 (2017), no. 4, 2357-2412, [arXiv:1605.03444].

[43] D. Grady, H. Sati, Twisted differential generalized cohomology theories and their Atiyah-Hirzebruch spectral sequence, [arXiv:1711.06650].

[44] D. Grady, H. Sati, Twisted smooth Deligne cohomology, Ann. Global Anal. Geom. 53 (2018), 445-466, [arXiv: 1706. 02742] [math.DG].

[45] R. Hain, Deligne-Beilinson cohomology of affine groups, [arXiv: 1507.03144].

[46] R. Harvey, H.B. Lawson, From sparks to grundles - differential characters, Comm. Anal. Geom. 14 (2006), no. 1, 25-58. 
[47] R. Harvey, H.B. Lawson, J. Zweck, The de Rham-Federer theory of differential characters and character duality, Amer. J. Math. 125 (2003), 791-847.

[48] N. Hitchin, Lectures on Special Lagrangian Submanifolds, AMS/IP Stud. Adv. Math., 23, Amer. Math. Soc., Providence, RI, (2001), 151-182.

[49] M.J. Hopkins, G. Quick, Hodge filtered complex bordism, J. Topol. 8 (2015), $147-183$.

[50] M.J. Hopkins, I.M. Singer, Quadratic functions in geometry, topology, and Mtheory, J. Differential Geom. 70 (2005), no. 3, 329-452.

[51] U. Jannsen, Deligne homology, Hodge-D-conjecture, and motives, Beilinson's Conjectures on Special Values of L-Functions, 305-372, Academic Press, Boston, MA, 1988.

[52] J.F. Jardine, Local Homotopy Theory, Springer, New York, 2015.

[53] A. Joyal, Notes on Logoi, http://www.math.uchicago.edu/ may/IMA/JOYAL/ Joyal.pdf.

[54] J.A. Lind, H. Sati, C. Westerland, Twisted iterated algebraic K-theory and topological T-duality for sphere bundles, [arXiv:1601.06285].

[55] J. Lott, Higher degree analogs of the determinant line bundle, Comm. Math. Phys. 230 (2002), 41-69.

[56] J. Lurie, Higher topos theory, Princeton University Press, Princeton, 2009.

[57] J. Lurie, Derived Algebraic Geometry IV: Deformation theory, http://www. math.harvard.edu/ lurie/papers/DAG-IV.pdf.

[58] J. Lurie, Higher Algebra, http://www.math.harvard.edu/ lurie/papers/ HA . pdf.

[59] V. Mathai, S. Wu, Analytic torsion for twisted de Rham complexes, J. Differential Geom. 88 (2011), no. 2, 297-332, [arXiv:0810.4204] [math.DG].

[60] J.P. May, $E_{\infty}$ Ring Spaces and $E_{\infty}$ Ring Spectra, with contributions by F. Quinn, N. Ray, and J. Tornehave, Springer-Verlag, Berlin, New York, 1977.

[61] L.G. Lewis, Jr., J.P. May, M. Steinberger, J.E. McClure, Equivariant Stable Homotopy Theory, Lecture Notes in Math., 1213, Springer-Verlag, Berlin, 1986.

[62] J.P. May, J. Sigurdsson, Parametrized Homotopy Theory, Amer. Math. Soc., Providence, RI, 2006.

[63] R. Picken, A cohomological description of abelian bundles and gerbes, Twenty Years of Bialowieza: A Mathematical Anthology, 217-228, World Sci. Monogr. Ser. Math., 8, World Sci. Publ., Hackensack, NJ, 2005.

[64] V.V. Prasolov, Elements of Combinatorial and Differential Topology, Amer. Math. Soc., Providence, RI, 2006.

[65] R. Rohm, E. Witten, The antisymmetric tensor field in superstring theory, Ann. Phys. 170 (1986), no. 2, 454-489.

[66] J. Rosenberg, Homological invariants of extensions of $C^{*}$-algebras, Operator Algebras and Applications, Part 1 (Kingston, Ont., 1980), 35-75, Proc. Sympos. Pure Math., 38, Amer. Math. Soc., Providence, RI, 1982.

[67] J. Rosenberg, Continuous-trace algebras from the bundle theoretic point of view, J. Aust. Math. Soc. Ser. A 47 (1989), 368-381. 
[68] H. Sati, A higher twist in string theory, J. Geom. Phys. 59 (2009), no. 3, 369-373, [arXiv:hep-th/0701232].

[69] H. Sati, Geometric and topological structures related to M-branes, Proc. Sympos. Pure Math., 81, 181-236, Amer. Math. Soc., Providence, RI, 2010.

[70] H. Sati, M-theory with framed corners and tertiary index invariants, SIGMA Symmetry Integrability Geom. Methods Appl. 10 (2014), 024, 28 pages, [arXiv:1203.4179] [hep-th].

[71] H. Sati, U. Schreiber, J. Stasheff, Differential twisted String- and Fivebrane structures, Comm. Math. Phys. 315 (2012), 169-213, [arXiv:0910.4001].

[72] H. Sati, C. Westerland, Twisted Morava K-theory and E-theory, J. Topol. 8 (2015), no. 4, 887-916, [arXiv:1109.3867] [math.AT].

[73] B. Shipley, HZZ-algebra spectra are differential graded algebras, Amer. J. Math. 129 (2007), no. 2, 351-379.

[74] U. Schreiber, Differential cohomology in a cohesive infinity-topos, [arXiv: 1310.7930] [math-ph].

[75] J. Simons, D. Sullivan, Axiomatic characterization of ordinary differential cohomology, J. Topol. 1 (2008), no. 1, 45-56.

[76] C. Teleman, K-theory and the moduli space of bundles on a surface and deformations of the Verlinde algebra, Topology, Geometry and Quantum Field Theory, 358-378, Cambridge Univ. Press, Cambridge, 2004.

[77] F. Thuillier, Deligne-Beilinson cohomology in $U(1)$ Chern-Simons theories, Mathematical Aspects of Quantum Field Theories, 233-271, Springer, Cham, 2015 .

[78] K. Waldorf, String connections and Chern-Simons theory, Trans. Amer. Math. Soc. 365 (2013), 4393-4432, [arXiv:0906.0117] [math.DG].

Daniel Grady djg9@nyu.edu

Mathematics Department, New York University Abu Dhabi, Saadiyat Island, Abu Dhabi, 129188, UAE

Hisham Sati hsati@nyu.edu

Mathematics Department, New York University Abu Dhabi, Saadiyat Island, Abu Dhabi, 129188, UAE 